\title{
A POINCARÉ-BIRKHOFF-WITT THEOREM FOR QUADRATIC ALGEBRAS WITH GROUP ACTIONS
}

\author{
ANNE V. SHEPLER AND SARAH WITHERSPOON
}

\begin{abstract}
Braverman, Gaitsgory, Polishchuk, and Positselski gave necessary and sufficient conditions for a nonhomogeneous quadratic algebra to satisfy the Poincaré-Birkhoff-Witt property when its homogeneous version is Koszul. We widen their viewpoint and consider a quotient of an algebra that is free over some (not necessarily semisimple) subalgebra. We show that their theorem holds under a weaker hypothesis: We require the homogeneous version of the nonhomogeneous quadratic algebra to be the skew group algebra (semidirect product algebra) of a finite group acting on a Koszul algebra, obtaining conditions for the Poincaré-Birkhoff-Witt property over (nonsemisimple) group algebras. We prove our main results by exploiting a double complex adapted from Guccione, Guccione, and Valqui (formed from a Koszul complex and a resolution of the group), giving a practical way to analyze Hochschild cohomology and deformations of skew group algebras in positive characteristic. We apply these conditions to graded Hecke algebras and Drinfeld orbifold algebras (including rational Cherednik algebras and symplectic reflection algebras) in arbitrary characteristic, with special interest in the case when the characteristic of the underlying field divides the order of the acting group.
\end{abstract}

\section{INTRODUCTION}

Poincaré-Birkhoff-Witt properties are used to isolate convenient canonical bases of algebras, identify algebras with formal deformations, and depict associated graded structures of algebras explicitly. They reveal how a set of generators for an ideal of relations defining an algebra may capture the graded structure implied by the entire ideal. Braverman and Gaitsgory [5] and Polishchuk and Positselski [22, 23] gave necessary and sufficient conditions for a nonhomogeneous quadratic algebra to satisfy the Poincaré-Birkhoff-Witt property when a homogeneous version is Koszul. Poincaré-Birkhoff-Witt theorems are often established by inspection of monomial orderings, noncommutative Gröbner bases, or other methods from noncommutative computational algebra. In contrast, Braverman and Gaitsgory used Hochschild cohomology to streamline arguments. They identified conditions that often provide an elegant alternative to direct application of Bergman's Diamond Lemma [3] (which can be tedious) for determining when a Poincaré-Birkhoff-Witt property holds. Thus their theory has been used widely to investigate various algebras in many settings. (See [23] and the references therein.)

Received by the editors September 25, 2012 and, in revised form, January 17, 2013.

2010 Mathematics Subject Classification. Primary 16S37, 16E40, 16S80, 16 S35.

Key words and phrases. Koszul algebras, skew group algebras, Hochschild cohomology, Drinfeld orbifold algebras.

The first author was partially supported by NSF grants \#DMS-0800951 and \#DMS-1101177.

The second author was partially supported by NSF grants \#DMS-0800832 and \#DMS1101399. 
Etingof and Ginzburg [8] noted that the conditions for a Poincaré-Birkhoff-Witt property developed by Braverman and Gaitsgory [5] and Polishchuk and Positselski [22,23] may be generalized by replacing the ground field $k$ by any semisimple ring (for example, a group ring $k G$ ) using the theory of Beilinson, Ginzburg, and Soergel [4] of Koszul rings. They exploited this generalization in investigations of symplectic reflection algebras. Later others applied it even more generally, for example, Halbout, Oudom, and Tang [16]. Yet the observation of Etingof and Ginzburg [8] does not apply in some interesting settings, in particular, when replacing the ground field by a ring that is not semisimple. For example, a theory of symplectic reflection algebras and graded Hecke algebras over fields of arbitrary characteristic would include exploration of quotient algebras over group $\operatorname{rings} k G$ when the characteristic of the field $k$ divides the order of the finite group $G$.

We show in Theorem 5.4 that the conditions of Braverman and Gaitsgory for a Poincaré-Birkhoff-Witt property hold over any finite group algebra (including nonsemisimple group algebras). We establish necessary and sufficient conditions for a filtered quadratic algebra over $k G$ to satisfy the PBW property when its homogeneous version is a skew group algebra (semidirect product algebra) formed from a finite group $G$ acting on a Koszul algebra $S$. The proof uses deformation theory and an explicit bimodule resolution $X_{\bullet}$. This resolution, defined in Section 4 and adapted from Guccione, Guccione, and Valqui [15], is composed of both the Koszul resolution for $S$ and the bar resolution for $k G$. Semisimplicity does not play a crucial role. However, there are potentially many graded deformations of the skew group algebra $S \# G$ in positive characteristic that this theory does not identify, corresponding to components of the resolution $X_{\bullet}$ that are not fully explored here. Other methods have been employed in the modular setting in some special cases; for example, see [14] for representation-theoretic techniques and [27] for an application of the Diamond Lemma.

We compare our techniques with those in the nonmodular setting: In characteristic zero, the group algebra $k G$ has trivial cohomology, and thus the Koszul resolution of $S$ is sufficient for analyzing the Hochschild cohomology of $S \# G$ (by a spectral sequence argument; see [9]). But in positive characteristic, the group algebra may exhibit nontrivial cohomology influencing the Hochschild cohomology of $S \# G$, and one seeks a more sophisticated replacement for the Koszul resolution of $S$ which nevertheless remains practical for determining concrete results (such as establishing a Poincaré-Birkhoff-Witt property). In this article, we highlight the complex $X_{\bullet}$ as a first tool in understanding the Hochschild cohomology of $S \# G$ in arbitrary characteristic and its deformation theory. Our general construction of $X \bullet$ in Section 4 takes as input arbitrary resolutions of $S$ and of $k G$ satisfying some natural conditions; the Koszul resolution of $S$ (in case $S$ is Koszul) and the bar resolution of $k G$ are but two of the potentially many useful choices. Our approach may lead to a better understanding of many algebras currently of interest, such as rational Cherednik algebras in positive characteristic. In Section 6 we illustrate these applications by focusing on a collection of such algebras, showing how known results in the modular setting may now be obtained directly from the methods of Braverman and Gaitsgory.

There are several papers containing generalizations of Koszul algebras for various purposes, those most relevant to our setting being [13, 19, 30]. We will not need these here, however, as the known properties of Koszul algebras over fields are 
sufficient to obtain our results. It would be interesting to determine whether it is possible to generalize the theory of Braverman and Gaitsgory more directly by using some equivalent definition of Koszul rings over (nonsemisimple) rings involving a bimodule complex (cf. [5, Proposition A.2(b)]).

Throughout this article, $k$ denotes a field (of arbitrary characteristic) and tensor symbols without subscript denote a tensor product over $k$ : $\otimes=\otimes_{k}$. (Tensor products over other rings will always be indicated). We assume all $k$-algebras have unity and all modules are left modules, unless otherwise specified. We use the notation $\mathbb{N}=\mathbb{Z}_{\geq 0}$, the nonnegative integers.

\section{Filtered And homogeneous Quadratic Algebras}

Quadratic algebras and their variations traditionally arise from taking a free algebra modulo a set of (nonhomogeneous) relations of degree two. Examples include symmetric algebras, commutative polynomial rings, skew/quantum polynomial rings, Weyl algebras, Clifford and exterior algebras, enveloping algebras of Lie algebras, and Sridharan enveloping algebras. But quadratic shape arises from any $\mathbb{N}$-graded algebra modulo an ideal generated in filtered degree two. Specifically, let $T=\bigoplus_{m} T^{m}$ be an $\mathbb{N}$-graded $k$-algebra. (We have in mind the tensor algebra (i.e., free algebra) $T_{k}(V)$ of a $k$-vector space $V$, or the tensor algebra $T_{B}(U)$ of a $B$-bimodule $U$ over a $k$-algebra $B$, for example, a group algebra.) Let $F^{m}(T)=T^{0} \oplus T^{1} \oplus \ldots \oplus T^{m}$ be the $m$-th filtered component of $T$ and fix a two-sided ideal $I$ of $T$.

A quotient $T / I$ is called a filtered quadratic algebra (or a nonhomogeneous quadratic algebra) if $I$ can be generated in filtered degree 2, i.e., if $I=\langle P\rangle$ for some $P \subset F^{2}(T)$. In this case, $P$ is called a set of filtered quadratic relations. Quadratic algebras are filtered algebras, with the $m$-th filtered component $F^{m}(T / I)=\left(F^{m}(T)+I\right) / I$ induced from that on $T$.

When a set of filtered quadratic relations $R$ resides exactly in the second graded component (homogeneous of degree 2), i.e., $R \subset T^{2}$, we call the quotient $T /\langle R\rangle$ a homogeneous quadratic algebra. Homogeneous quadratic algebras are not only filtered, but also graded: $T / I=\bigoplus_{m}\left(T^{m}+I\right) / I$ for $I=\langle R\rangle$.

One may easily associate to every filtered quadratic algebra $T / I$ two different graded versions. We might cross out lower order terms in a generating set of relations for the algebra, or, instead, cross out lower order terms in each element of the entire ideal of relations. The Poincaré-Birkhoff-Witt conditions are precisely those under which these two graded versions of the original algebra coincide, as we now recall.

On one hand, we may simply ignore those parts of each defining relation in $P$ of degree less than two to obtain a simplified version of the original filtered algebra which is homogeneous quadratic. Formally, we let $\pi$ denote projection onto the second graded component of the tensor algebra: $\pi: T \rightarrow T^{2}$. If $P$ is a set of filtered quadratic relations defining the algebra $T / I$, then $\pi(P)$ defines a graded quadratic algebra

$$
T /\langle\pi(P)\rangle,
$$

called the homogeneous quadratic algebra determined by $P$ (or the induced homogeneous quadratic algebra). Although this construction depends on the choice of generators $P$ for $I$, often a choice can be made to capture the degree two aspect of the entire ideal of relations. 
On the other hand, we may consider the traditional associated graded algebra

$$
\operatorname{gr}(T / I)=\bigoplus_{m} \mathcal{F}_{m} / \mathcal{F}_{m-1},
$$

where $\mathcal{F}_{m}=F^{m}(T / I)$. This graded version of the original algebra does not depend on the choice of generators $P$ of the ideal $I$ of relations. We realize the associated graded algebra concretely also as a homogeneous version of the original filtered quadratic algebra by projecting each element in the ideal $I$ onto its leading homogeneous part and taking the quotient by the resulting ideal: The associated homogeneous quadratic algebra (also called the leading homogeneous algebra) is defined as

$$
T /\langle\mathrm{LH}(I)\rangle,
$$

where $\operatorname{LH}(I)=\{\mathrm{LH}(f): f \in I\}$ and $\operatorname{LH}(f)$ picks off the highest homogeneous part of $f$ in $T$. (For $f=\sum_{i=0}^{d} f_{i}$ with each $f_{i}$ in $T^{i}$ and $f_{d}$ nonzero, $\operatorname{LH}(f)=f_{d}$, and $\mathrm{LH}(0)=0$.) This associated homogeneous quadratic algebra is isomorphic as a graded $k$-algebra to the associated graded algebra (see Li [18, Theorem 3.2]):

$$
\operatorname{gr}(T / I) \cong T /\langle\mathrm{LH}(I)\rangle \text {. }
$$

We say the original filtered quadratic algebra has Poincaré-Birkhoff-Witt type when the associated homogeneous quadratic algebra and the homogeneous quadratic algebra determined by $P$ coincide, and thus both give the associated graded algebra. (This terminology arises in analogy with the original PBW Theorem for universal enveloping algebras of Lie algebras.) More precisely, let

$$
\phi: T \rightarrow \operatorname{gr}(T / I)
$$

be the natural $k$-algebra epimorphism. Then (again, see Li [18, Theorem 3.2])

$$
\operatorname{Ker} \phi=\langle\operatorname{LH}(I)\rangle,
$$

which contains $\langle\pi(P)\rangle$ when $I=\langle P\rangle$. Thus, a natural surjection $p$ always arises from the homogeneous quadratic algebra determined by $P$ to the associated graded algebra of the filtered quadratic algebra:

$$
p: T /\langle\pi(P)\rangle \rightarrow \operatorname{gr}(T /\langle P\rangle) .
$$

A filtered quadratic algebra exhibits $\mathbf{P B W}$ type (with respect to $P$ ) exactly when it can be written as $T /\langle P\rangle$ for some set of filtered quadratic relations $P$ with $p$ an isomorphism of graded algebras, i.e., when

$$
\langle\pi(P)\rangle=\operatorname{Ker} \phi=\langle\operatorname{LH}(I)\rangle,
$$

and thus the homogeneous versions of the filtered quadratic algebra all coincide:

$$
T /\langle\pi(P)\rangle \cong \operatorname{gr}(T /\langle P\rangle) \cong T /\langle\mathrm{LH}\langle P\rangle\rangle .
$$

In this case, we say that $P$ is a set of $\mathbf{P B W}$ generating relations.

The definition of PBW type depends on $P$, as we see in Example 2.3 below. But if $T^{0}=k$, we may require (without loss of generality) that $P$ be a $k$-subspace, in which case a set of PBW generating relations $P$ is unique. In fact, a set of PBW generating relations is always unique up to additive closure over the degree zero component of $T$, as we explain in the next proposition. 
Proposition 2.1. $P B W$ filtered quadratic algebras have unique PBW filtered quadratic relations up to addition and multiplication by degree zero elements: If $P, P^{\prime}$ are each $P B W$ filtered quadratic relations defining the same filtered quadratic algebra $T / I$ (that is, $\langle P\rangle=I=\left\langle P^{\prime}\right\rangle$ ), then $P$ and $P^{\prime}$ generate the same $T^{0}$-bimodule. Thus, if both $P$ and $P^{\prime}$ are closed under addition and under multiplication by degree zero elements in $T$, then $P=P^{\prime}$.

Proof. We check that for any set $P$ of PBW relations,

$$
\langle P\rangle \cap F^{1}(T)=\{0\} \quad \text { and } \quad\langle P\rangle \cap F^{2}(T)=T^{0} P T^{0},
$$

where $T^{0} P T^{0}$ is the $T^{0}$-bimodule generated by $P$. The first claim is immediate: Since $\pi(P) \subset T^{2}$, the algebra $T /\langle\pi(P)\rangle$ contains $F^{1}(T)$ as a subspace; if $\langle P\rangle \cap$ $F^{1}(T) \neq\{0\}$, then $\operatorname{gr}(T /\langle P\rangle)$ does not contain $F^{1}(T)$ as a subspace, and so $p$ cannot be an isomorphism.

Now let $x$ be any element of $\langle P\rangle \cap F^{2}(T)$ with $x \notin T^{0} P T^{0}$. Note that for all $y \in T^{0} P T^{0}, \pi(x) \neq \pi(y)$. Otherwise, some nonzero $x-y$ would lie in $F^{1}(T)$ (as $\pi(x-y)=0)$, implying that $\langle P\rangle \cap F^{1}(T)$ is nonzero. Hence $\pi(x) \notin \pi\left(T^{0} P T^{0}\right)=$ $T^{0} \pi(P) T^{0}$. But this contradicts the PBW property, which implies that $\pi(x) \in$ $\langle\mathrm{LH}\langle P\rangle\rangle=\langle\pi(P)\rangle$ (a homogeneous ideal) and hence $\pi(x) \in T^{0} \pi(P) T^{0}$.

Lastly, if $P$ and $P^{\prime}$ are both PBW filtered quadratic relations generating the ideal $I$, then $T^{0} P T^{0}=I \cap F^{2}(T)=T^{0} P^{\prime} T^{0}$, and $P$ and $P^{\prime}$ generate the same $T^{0}$-bimodule.

Example 2.3. We give an example of a filtered quadratic algebra that exhibits PBW type with respect to one generating set of relations but not another. Let $V=\operatorname{Span}_{k}\{x, y\}$ and let $I$ be the two-sided ideal in the tensor algebra $T=T(V)$ (over $k$ ) generated by $P=\operatorname{Span}_{k}\{x y-x, y x-y\}$ (we suppress tensor signs in $T$ ). The filtered quadratic algebra $T(V) / I=T(V) /\langle P\rangle$ is not of PBW type with respect to $P$. Indeed, the graded summand of the associated graded algebra of degree 2 has dimension 0 over $k$ since a quick calculation verifies that $x^{2}-x$ and $y^{2}-y$ both lie in the ideal $\langle P\rangle$, implying that all of $T^{2}(V)=V \otimes V$ projects to zero in $T(V) /\langle P\rangle$ after passing to the associated graded algebra. But $\pi(P)=\operatorname{Span}_{k}\{x y, y x\} \subset V \otimes V$ defines a homogeneous quadratic algebra $T(V) /\langle x y, y x\rangle$ whose graded summand of degree 2 has dimension 2 over $k$ (with basis $\left\{x^{2}, y^{2}\right\}$ ). Note that the filtered quadratic algebra $T(V) / I$ exhibits PBW type with respect to a different set of generators for the ideal $I$. If we extend $P$ to

$$
P^{\prime}=\operatorname{Span}_{k}\left\{x y-x, y x-y, x^{2}-x, y^{2}-y\right\},
$$

then $T(V) / I$ is of PBW type with respect to $P^{\prime}$, as its associated graded algebra is isomorphic to

$$
T(V) /\left\langle x y, y x, x^{2}, y^{2}\right\rangle \cong T(V) /\left\langle\pi\left(P^{\prime}\right)\right\rangle
$$

In Section 5 we will analyze filtered and homogeneous quadratic algebras when $T$ is a free algebra. Traditional quadratic algebras arise as quotients of a tensor algebra $T$ of a $k$-vector space $V$. We expand this view and consider (more generally) bimodules $V$ over an arbitrary $k$-algebra $B$ in order to include constructions of algebras of quadratic shape naturally appearing in other settings. First we recall Hochschild cohomology and deformations in the next section, and construct the needed resolution $X \bullet$ in Section 4 . 


\section{Deformations and Koszul algebras}

Let $A$ be a $k$-algebra. Let $M$ be an $A$-bimodule, equivalently, an $A^{e}$-module, where $A^{e}=A \otimes A^{o p}$, the enveloping algebra of $A$. As $k$ is a field, the Hochschild cohomology of $M$ is

$$
\operatorname{HH}^{n}(A, M)=\operatorname{Ext}_{A^{e}}^{n}(A, M) .
$$

This cohomology can be examined explicitly using the bar resolution, a free resolution of the $A^{e}$-module $A$ :

$$
\cdots \stackrel{\delta_{3}}{\longrightarrow} A \otimes A \otimes A \otimes A \stackrel{\delta_{2}}{\longrightarrow} A \otimes A \otimes A \stackrel{\delta_{1}}{\longrightarrow} A \otimes A \stackrel{\delta_{0}}{\longrightarrow} A \rightarrow 0,
$$

where

$$
\delta_{n}\left(a_{0} \otimes \cdots \otimes a_{n+1}\right)=\sum_{i=0}^{n}(-1)^{i} a_{0} \otimes \cdots \otimes a_{i} a_{i+1} \otimes \cdots \otimes a_{n+1}
$$

for all $n \geq 0$ and $a_{0}, \ldots, a_{n+1}$ in $A$. If $M=A$, we abbreviate, $\operatorname{HH}^{n}(A):=$ $\mathrm{HH}^{n}(A, A)$.

A deformation of $A$ over $k[t]$ is an associative $k[t]$-algebra with underlying vector space $A[t]$ and multiplication determined by

$$
a_{1} * a_{2}=a_{1} a_{2}+\mu_{1}\left(a_{1} \otimes a_{2}\right) t+\mu_{2}\left(a_{1} \otimes a_{2}\right) t^{2}+\cdots,
$$

where $a_{1} a_{2}$ is the product of $a_{1}$ and $a_{2}$ in $A$ and each $\mu_{j}: A \otimes A \rightarrow A$ is a $k$ linear map. (Only finitely many terms are nonzero for each pair $a_{1}, a_{2}$ in the above expansion.)

We record some needed properties of $\mu_{1}$ and $\mu_{2}$. Note that $\operatorname{Hom}_{k}(A \otimes A, A) \cong$ Hom $_{A^{e}}(A \otimes A \otimes A \otimes A, A)$ since the $A^{e}$-module $A \otimes A \otimes A \otimes A$ is (tensor) induced from the $k$-module $A \otimes A$, and we identify $\mu_{1}$ with a 2 -cochain on the bar resolution (3.1). Associativity of $*$ implies that $\mu_{1}$ is a Hochschild 2-cocycle, i.e., that

$$
a_{1} \mu_{1}\left(a_{2} \otimes a_{3}\right)+\mu_{1}\left(a_{1} \otimes a_{2} a_{3}\right)=\mu_{1}\left(a_{1} a_{2} \otimes a_{3}\right)+\mu_{1}\left(a_{1} \otimes a_{2}\right) a_{3}
$$

for all $a_{1}, a_{2}, a_{3} \in A$, or, equivalently, that $\delta_{3}^{*}\left(\mu_{1}\right)=0$ : One need only expand each side of the equation $a_{1} *\left(a_{2} * a_{3}\right)=\left(a_{1} * a_{2}\right) * a_{3}$ and compare coefficients of $t$. Comparing coefficients of $t^{2}$ instead yields

$$
\delta_{3}^{*}\left(\mu_{2}\right)\left(a_{1} \otimes a_{2} \otimes a_{3}\right)=\mu_{1}\left(a_{1} \otimes \mu_{1}\left(a_{2} \otimes a_{3}\right)\right)-\mu_{1}\left(\mu_{1}\left(a_{1} \otimes a_{2}\right) \otimes a_{3}\right)
$$

for all $a_{1}, a_{2}, a_{3} \in A$. Thus we consider $\mu_{2}$ to be a cochain on the bar resolution whose coboundary is given as above. Generally, for all $i \geq 1$,

$$
\delta_{3}^{*}\left(\mu_{i}\right)\left(a_{1} \otimes a_{2} \otimes a_{3}\right)=\sum_{j=1}^{i-1} \mu_{j}\left(\mu_{i-j}\left(a_{1} \otimes a_{2}\right) \otimes a_{3}\right)-\mu_{j}\left(a_{1} \otimes \mu_{i-j}\left(a_{2} \otimes a_{3}\right)\right) .
$$

We call the right side of the last equation the $(i-1)$-th obstruction.

Now we recall graded deformations. Assume that the algebra $A$ is $\mathbb{N}$-graded. Let $t$ be an indeterminate and extend the grading to $A[t]$ by assigning $\operatorname{deg} t=1$. A graded deformation of $A$ over $k[t]$ is a deformation $A_{t}$ of $A$ which is also graded; each map $\mu_{j}: A \otimes A \rightarrow A$ is necessarily homogeneous of degree $-j$ in this case. An $i$-th level graded deformation of $A$ is a graded associative $k[t] /\left(t^{i+1}\right)$ algebra $A_{i}$ whose underlying vector space is $A[t] /\left(t^{i+1}\right)$ and whose multiplication is determined by

$$
a_{1} * a_{2}=a_{1} a_{2}+\mu_{1}\left(a_{1} \otimes a_{2}\right) t+\mu_{2}\left(a_{1} \otimes a_{2}\right) t^{2}+\cdots+\mu_{i}\left(a_{1} \otimes a_{2}\right) t^{i}
$$


for some maps $\mu_{j}: A \otimes A \rightarrow A$ extended to be linear over $k[t] /\left(t^{i+1}\right)$. We call $\mu_{j}$ the $j$-th multiplication map of the deformation $A_{i}$; note that it is homogeneous of degree $-j$ as $A_{i}$ is graded. We say that an $(i+1)$-st level graded deformation $A_{i+1}$ of $A$ extends (or lifts) an $i$-th level graded deformation $A_{i}$ of $A$ if the $j$-th multiplication maps agree for all $j \leq i$.

For any $\mathbb{N}$-graded $A$-bimodule $M$, the bar resolution (3.1) induces a grading on Hochschild cohomology $\operatorname{HH}^{n}(A, M)$ in the following way: Let

$$
\operatorname{Hom}_{k}^{i}\left(A^{\otimes n}, M\right)
$$

be the space of all homogeneous $k$-linear maps from $A^{\otimes n}$ to $M$ of degree $i$, i.e., mapping the degree $j$ component of $A^{\otimes n}$ (for any $j$ ) to the degree $j+i$ component of $M$, where $\operatorname{deg}\left(a_{1} \otimes \cdots \otimes a_{n}\right)$ in $A^{\otimes n}$ is $\operatorname{deg} a_{1}+\ldots+\operatorname{deg} a_{n}$ for $a_{1}, \ldots, a_{n}$ homogeneous in $A$. The differential on the bar complex (3.1) has degree zero, so the spaces of coboundaries and cocycles inherit this grading. We denote the resulting $i$-th graded component of $\operatorname{HH}^{n}(A, M)$ by $\mathrm{HH}^{n, i}(A, M)$, so that

$$
\mathrm{HH}^{n}(A, M)=\bigoplus_{i} \mathrm{HH}^{n, i}(A, M) \text {. }
$$

The following proposition is a consequence of [5, Proposition 1.5].

Proposition 3.6. First level graded deformations of the algebra $A$ define elements of the Hochschild cohomology space $\mathrm{HH}^{2,-1}(A)$. Two such deformations are (graded) isomorphic if, and only if, the corresponding cocycles are cohomologous. All obstructions to lifting an $(i-1)$-th level graded deformation to the next level lie in $\mathrm{HH}^{3,-i}(A)$; an $(i-1)$-th level deformation lifts to the $i$-th level if and only if its $(i-1)$-th obstruction cocycle is zero in cohomology.

We are most interested in deformations of skew group algebras formed from Koszul algebras with group actions, and we recall Koszul algebras (over the field $k$ ) next. Let $V$ be a finite dimensional vector space. Let

$$
T_{k}(V)=k \oplus V \oplus(V \otimes V) \oplus(V \otimes V \otimes V) \oplus \ldots,
$$

the tensor algebra of $V$ over $k$ with $i$-th graded piece $T_{k}^{i}(V):=V^{\otimes i}$ and $T_{k}^{0}(V)=k$.

Let $R$ be a subspace of $T_{k}^{2}(V)$, and let $S=T_{k}(V) /\langle R\rangle$ be the corresponding homogeneous quadratic algebra. Let $K^{0}(S)=k, K^{1}(S)=V$, and for each $n \geq 2$, define

$$
K^{n}(S)=\bigcap_{j=0}^{n-2}\left(V^{\otimes j} \otimes R \otimes V^{\otimes(n-2-j)}\right) .
$$

Set

$$
\widetilde{K}^{n}(S)=S \otimes K^{n}(S) \otimes S .
$$

Then $\widetilde{K}^{0}(S) \cong S \otimes S, \widetilde{K}^{1}(S)=S \otimes V \otimes S, \widetilde{K}^{2}(S)=S \otimes R \otimes S$, and, in general, $\widetilde{K}^{n}(S)$ embeds as an $S^{e}$-submodule into $S^{\otimes(n+2)}$, the $n$-th component of the bar resolution (3.1). We apply the differential $\delta_{n}$, defined in (3.2), to an element in $\widetilde{K}^{n}(S)$ and note that the terms indexed by $i=1,2, \ldots, n-1$ vanish (due to the factors in the space of relations $R$ ). The remaining terms are clearly in $\widetilde{K}^{n-1}(S)$. We may thus consider $\widetilde{K}^{\bullet}(S)$ to be a complex with differential $d$ (restricted from the bar complex, $\left.d_{n}:=\left.\delta_{n}\right|_{\widetilde{K}^{n}(S)}\right)$, called the Koszul complex:

$$
\ldots \stackrel{d_{3}}{\longrightarrow} \widetilde{K}^{2}(S) \stackrel{d_{2}}{\longrightarrow} \widetilde{K}^{1}(S) \stackrel{d_{1}}{\longrightarrow} \widetilde{K}^{0}(S) \stackrel{d_{0}}{\longrightarrow} S \rightarrow 0 .
$$


By definition, $S$ is a Koszul algebra if the related complex $\bar{K}^{\bullet}(S)$, defined by $\bar{K}^{n}(S):=S \otimes K^{n}(S)$, is a resolution of the $S$-module $k$ on which each element of $V$ acts as 0 . (Note that $\bar{K}^{n}(S) \cong \widetilde{K}^{n}(S) \otimes_{S} k$; differentials are $d_{n} \otimes$ id .) It is well known that $S$ is a Koszul algebra if, and only if, $\widetilde{K}^{\bullet}(S)$ is a resolution of the $S^{e}$-module $S$. (See e.g. [5, Proposition A.2] or [17].)

\section{Resolutions FOR SKEW GROUP ALGEBRAS}

In this section, we consider any finite group $G$ and any $k$-algebra $S$ upon which $G$ acts by automorphisms. We work in arbitrary characteristic to develop techniques helpful in the modular setting. We explain how to construct a resolution of the skew group algebra $A=S \# G$ from resolutions of $S$ and of $k G$, generalizing a construction of Guccione, Guccione, and Valqui [15, §4.1]. It should be compared to the double complexes in [25, $§ 2.2]$ and more generally in [28, Corollary 3.4] that are used to build spectral sequences. We apply the resolution in the next section to generalize the result of Braverman and Gaitsgory [5, Theorem 4.1] on the Poincaré-Birkhoff-Witt property. Some of our assumptions in this section will seem restrictive, however the large class of examples to which we generalize their result in Theorem [5.4 all satisfy the assumptions, as do the modular versions of Drinfeld orbifold algebras, graded Hecke algebras, and symplectic reflection algebras in Section 6 .

In characteristic zero, $\mathrm{HH}^{\bullet}(S \# G) \cong \mathrm{HH}^{\bullet}(S, S \# G)^{G}$ as a consequence of a spectral sequence argument, and the latter may be obtained from a resolution of $S$. (In the special case that $S$ is Koszul, one thus has a convenient resolution at hand for analyzing the cohomology of $S \# G$ and its deformation theory.) But in the modular setting, when the characteristic of $k$ divides the order of $G$, the spectral sequence no longer merely produces $G$-invariants (as the group may have nontrivial cohomology), and thus this technique for simplifying the cohomology of the skew group algebra fails. The resolution of $S \# G$ constructed in this section retains some of the flavor of the Koszul resolution of $S$ so as to allow similar Koszul techniques to be applied in the modular setting.

We first recall the definition of a skew group algebra. The skew group algebra $S \# G$ is a semidirect product algebra: It is the $k$-vector space $S \otimes k G$ together with multiplication given by $(r \otimes g)(s \otimes h)=r\left({ }^{g} s\right) \otimes g h$ for all $r, s$ in $S$ and $g, h$ in $G$, where ${ }^{g} s$ is the image of $s$ under the automorphism $g$. (We are most interested in skew group algebras arising from the linear action of $G$ on a finite dimensional vector space $V$ and its induced actions on tensor algebras, symmetric algebras, Koszul algebras, and homogeneous quadratic algebras all generated by $V$.)

We need the notion of Yetter-Drinfeld modules: A vector space $V$ is a YetterDrinfeld module over $G$ if $V$ is $G$-graded, that is, $V=\bigoplus_{g \in G} V_{g}$, and $V$ is a $k G$-module with $h\left(V_{g}\right)=V_{h g h^{-1}}$ for all $g, h \in G$. Any $k G$-module $V$ is trivially a Yetter-Drinfeld module by letting $V_{1}=V$ and $V_{g}=0$ for all nonidentity group elements $g$. Similarly, any algebra $S$ on which $G$ acts by automorphisms is a Yetter-Drinfeld module in this way. Alternatively, the group algebra $k G$ itself may be considered to be a Yetter-Drinfeld module by letting the $g$-component be all scalar multiples of $g$, for each $g \in G$, and by letting $G$ act on $k G$ by conjugation. The skew group algebra $A=S \# G$ is a Yetter-Drinfeld module by combining these 
two structures:

$$
A=\bigoplus_{g \in G} A_{g}
$$

where $A_{g}=S g$ and $G$ acts on $A$ by conjugation (an inner action). A morphism of Yetter-Drinfeld modules is a $k G$-module homomorphism that preserves the $G$ grading. We use a braiding on the category of Yetter-Drinfeld modules: Given any two Yetter-Drinfeld modules $V, W$ over $G$, their tensor product $V \otimes W$ is again a Yetter-Drinfeld module with

$$
(V \otimes W)_{g}=\bigoplus_{x y=g}\left(V_{x} \otimes W_{y}\right)
$$

and the usual $G$-action on a tensor product: ${ }^{g}(v \otimes w)={ }^{g} v \otimes{ }^{g} w$ for all $g \in G$, $v \in V, w \in W$. There is an isomorphism of Yetter-Drinfeld modules

$$
c_{V, W}: V \otimes W \rightarrow W \otimes V
$$

given by $c_{V, W}(v \otimes w)={ }^{g} w \otimes v$ for all $v \in V_{g}, w \in W, g \in G$, called the braiding.

We will now construct an $A^{e}$-free resolution of $A=S \# G$ from a $(k G)^{e}$-free resolution of $k G$ and an $S^{e}$-free resolution of $S$. First let

$$
\cdots \rightarrow C_{1} \rightarrow C_{0} \rightarrow k G \rightarrow 0
$$

be a $(k G)^{e}$-free resolution of $k G$. We assume that each $C_{i}$ is $G$-graded with grading preserved by the bimodule structure, that is, $g\left(\left(C_{i}\right)_{h}\right) l=\left(C_{i}\right)_{g h l}$ for all $g, h, l$ in $G$. Note this implies that $C_{i}$ is a Yetter-Drinfeld module where ${ }^{g} c=g c g^{-1}$ for all $g$ in $G$ and $c$ in $C_{i}$. We also assume that the differentials preserve the $G$-grading. Assume that as a free $(k G)^{e}$-module, $C_{i}=k G \otimes C_{i}^{\prime} \otimes k G$ for a $G$-graded vector space $C_{i}^{\prime}$ whose $G$-grading induces that on $C_{i}$ under the usual tensor product of $G$-graded vector spaces. For example, the bar resolution of $k G$ satisfies all these properties. Another instance can be found in Example 4.6 below.

Next let

$$
\cdots \rightarrow D_{1} \rightarrow D_{0} \rightarrow S \rightarrow 0
$$

be an $S^{e}$-free resolution of $S$ consisting of left $k G$-modules for which the differentials are $k G$-module homomorphisms, and the left actions of $S$ and of $k G$ are compatible in the sense that they induce a left action of $A=S \# G$. We consider each $D_{i}$ to be a Yetter-Drinfeld module by setting it all in the component of the identity. Write the free $S^{e}$-module $D_{i}$ as $S \otimes D_{i}^{\prime} \otimes S$, for a vector space $D_{i}^{\prime}$. For example, the bar resolution of $S$ satisfies these properties under the usual action of $G$ on tensor products. If $S$ is a Koszul algebra on which $G$ acts by graded automorphisms, the Koszul resolution of $S$ also satisfies these properties.

We now induce both $C_{\bullet}$ and $D_{\bullet}$ to $A$ by tensoring with $A$ in each degree. We induce $C$. from $k G$ to $A$ on the left: Since $A$ is free as a right $k G$-module under multiplication, and $A \otimes_{k G} k G \cong A$, we obtain an exact sequence of $A \otimes(k G)^{o p_{-}}$ modules,

$$
\cdots \rightarrow A \otimes_{k G} C_{1} \rightarrow A \otimes_{k G} C_{0} \rightarrow A \rightarrow 0 .
$$

Similarly, we induce $D_{\bullet}$ from $S$ to $A$ on the right: Since $A$ is free as a left $S$-module, and $S \otimes_{S} A \cong A$,

$$
\cdots \rightarrow D_{1} \otimes_{S} A \rightarrow D_{0} \otimes_{S} A \rightarrow A \rightarrow 0
$$

is an exact sequence of $S \otimes A^{o p}$-modules.

We extend the actions on the modules in each of these two sequences so that they become sequences of $A^{e}$-modules. This will allow us to take their tensor product 
over $A$. We extend the right $k G$-module structure on $A \otimes_{k G} C \bullet$ to a right $A$-module structure by using the braiding to define a right action of $S$ : For all $a \in A, g \in G$, $x \in\left(C_{i}\right)_{g}, s \in S$, we set

$$
(a \otimes x) \cdot s:=a\left({ }^{g} s\right) \otimes x .
$$

We combine this right action of $S$ with the right action of $k G$. Under our assumptions, this results in a right action of $A$ on $A \otimes_{k G} C_{i}$. To see that it is well defined, note that if $h \in G$, then $(a h \otimes x) \cdot s=a h\left({ }^{g} s\right) \otimes x=a\left({ }^{h g} s\right) \otimes h x=(a \otimes h x) \cdot s$. Thus $A \otimes_{k G} C_{i}$ is an $A$-bimodule and the action commutes with the differentials by the assumption that the differentials on $C$. preserve the $G$-grading.

We extend the left $S$-module structure on $D_{i} \otimes_{S} A$ to a left $A$-module structure by defining a left action of $k G$ :

$$
g \cdot(y \otimes a):={ }^{g} y \otimes g a
$$

for all $g \in G, y \in D_{i}, a \in A$. It is well defined since $g s=\left({ }^{g} s\right) g$ for all $s \in S$, and indeed gives a left action of $A$ on $D_{i} \otimes_{S} A$. Again this action commutes with the differentials by our assumption that the differentials on $D_{\bullet}$ are $k G$-module homomorphisms.

We use the $A$-bimodule structures on $A \otimes_{k G} C \bullet$ and on $D_{\bullet} \otimes_{S} A$ defined above and consider each as a complex of $A^{e}$-modules. (Note that we have not assumed they consist of projective $A^{e}$-modules.) We take their tensor product over $A$, setting $X_{\bullet \bullet \bullet}:=\left(A \otimes_{k G} C_{\bullet}\right) \otimes_{A}\left(D_{\bullet} \otimes_{S} A\right)$. That is, for all $i, j \geq 0$,

$$
X_{i, j}:=\left(A \otimes_{k G} C_{i}\right) \otimes_{A}\left(D_{j} \otimes_{S} A\right),
$$

with horizontal and vertical differentials

$$
d_{i, j}^{h}: X_{i, j} \rightarrow X_{i-1, j} \quad \text { and } \quad d_{i, j}^{v}: X_{i, j} \rightarrow X_{i, j-1}
$$

given by $d_{i, j}^{h}:=d_{i} \otimes$ id and $d_{i, j}^{v}:=(-1)^{i}$ id $\otimes d_{j}$, where $d_{i}$ and $d_{j}$ denote the

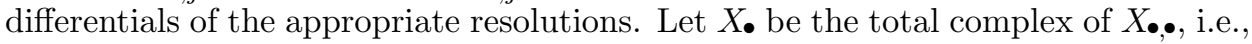
the complex

$$
\cdots \rightarrow X_{2} \rightarrow X_{1} \rightarrow X_{0} \rightarrow A \rightarrow 0
$$

with $X_{n}=\bigoplus_{i+j=n} X_{i, j}$.

Theorem 4.3. Let $S$ be a k-algebra with action of a finite group $G$ by automorphisms and set $A=S \# G$. Let $X \bullet$ be the complex defined in (4.2) from factors $C_{i}=k G \otimes C_{i}^{\prime} \otimes k G$ and $D_{i}=S \otimes D_{i}^{\prime} \otimes S$ as above. Then $X_{\bullet}$ is a free resolution of the $A^{e}$-module $A$, and for each $i, j$, the $A^{e}$-module $X_{i, j}$ is isomorphic to $A \otimes C_{i}^{\prime} \otimes D_{j}^{\prime} \otimes A$.

In the case that $C_{\bullet}$ is the (normalized) bar resolution of $k G$ and $D_{\bullet}$ is the Koszul resolution of $S(V)$, our resolution $X_{\bullet}$ is precisely that in [15, §4].

Proof. We first check that for each $i, j$, the $A^{e}$-module $X_{i, j}$ is free. By construction,

$$
\begin{aligned}
X_{i, j} & =\left(A \otimes_{k G} k G \otimes C_{i}^{\prime} \otimes k G\right) \otimes_{A}\left(S \otimes D_{j}^{\prime} \otimes S \otimes_{S} A\right) \\
& \cong\left(A \otimes C_{i}^{\prime} \otimes k G\right) \otimes_{A}\left(S \otimes D_{j}^{\prime} \otimes A\right) .
\end{aligned}
$$

We claim that this is isomorphic to $A \otimes C_{i}^{\prime} \otimes D_{j}^{\prime} \otimes A$ as an $A^{e}$-module. To see this, first define a map as follows:

$$
\begin{aligned}
\left(A \otimes C_{i}^{\prime} \otimes k G\right) \times\left(S \otimes D_{j}^{\prime} \otimes A\right) & \rightarrow A \otimes C_{i}^{\prime} \otimes D_{j}^{\prime} \otimes A \\
(a \otimes x \otimes g, s \otimes y \otimes b) & \mapsto a\left({ }^{h g} s\right) \otimes x \otimes{ }^{g} y \otimes g b,
\end{aligned}
$$


where $x \in\left(C_{i}^{\prime}\right)_{h}$. This map is bilinear by its definition, and we check that it is $A$-balanced: If $r \in S, \ell \in G$, then on the one hand,

$$
\begin{aligned}
((a \otimes x \otimes g) \cdot(r \ell), s \otimes y \otimes b) & =\left(a\left({ }^{h g} r\right) \otimes x \otimes g \ell, s \otimes y \otimes b\right) \\
& \mapsto a\left({ }^{h g} r\right)\left({ }^{h g \ell} s\right) \otimes x \otimes{ }^{g \ell} y \otimes g \ell b,
\end{aligned}
$$

while on the other hand,

$$
\begin{aligned}
((a \otimes x \otimes g),(r \ell) \cdot(s \otimes y \otimes b)) & =\left(a \otimes x \otimes g, r\left({ }^{\ell} s\right) \otimes{ }^{\ell} y \otimes \ell b\right) \\
& \left.\mapsto a\left({ }^{h g} r\right)\left({ }^{h g \ell} s\right) \otimes x \otimes{ }^{g \ell} y \otimes g \ell b\right) .
\end{aligned}
$$

Therefore there is an induced map

$$
\left(A \otimes C_{i}^{\prime} \otimes k G\right) \otimes_{A}\left(S \otimes D_{j}^{\prime} \otimes A\right) \rightarrow A \otimes C_{i}^{\prime} \otimes D_{j}^{\prime} \otimes A .
$$

It is straightforward to verify that an inverse map is given by

$$
a \otimes x \otimes y \otimes b \mapsto(a \otimes x \otimes 1) \otimes(1 \otimes y \otimes b) .
$$

Therefore the two spaces are isomorphic as claimed.

We wish to apply the Künneth Theorem, and to that end we check that each term in the complex $A \otimes_{k G} C$. consists of free right $A$-modules and that the image of each differential in the complex is projective as a right $A$-module. This may be proved inductively, starting on one end of the complex:

$$
\cdots \stackrel{f_{2}}{\longrightarrow} A \otimes_{k G} C_{1} \stackrel{f_{1}}{\longrightarrow} A \otimes_{k G} C_{0} \stackrel{f_{0}}{\longrightarrow} A \rightarrow 0 .
$$

To see directly that each $A \otimes_{k G} C_{i}$ is free as a right $A$-module, write

$$
A \otimes_{k G} C_{i}=A \otimes_{k G}\left(k G \otimes C_{i}^{\prime} \otimes k G\right) \cong A \otimes C_{i}^{\prime} \otimes k G .
$$

Choose a $k$-linear finite basis $\left\{x_{m} \mid 1 \leq m \leq r_{i}\right\}$ of $C_{i}^{\prime}$ for which each $x_{m}$ is homogeneous with respect to the $G$-grading, and $r_{i}=\operatorname{dim}_{k} C_{i}^{\prime}$. (A similar idea works if the $C_{i}^{\prime}$ are infinite dimensional; however, since $G$ is finite, it is reasonable to assume that the $C_{i}^{\prime}$ are finite dimensional.) Then a set of free generators of $A \otimes_{k G} C_{i}$ as a right $A$-module is

$$
\left\{g \otimes x_{m} \otimes 1 \mid g \in G, 1 \leq m \leq r_{i}\right\} .
$$

Indeed, if we fix $g$ in $G$ and $x_{m}$ as above, with $x_{m}$ in the $\ell$-component $(\ell \in G)$, then for each $s$ in $S$ and $h$ in $G$,

$$
\left(g \otimes x_{m} \otimes 1\right) \cdot\left(\ell^{-1} g^{-1} s\right) h=s g \otimes x_{m} \otimes h,
$$

and consequently the full subspace $S g \otimes x_{m} \otimes k G$ is generated from this single element. It also follows that they are independent. Since $A$ is right $A$-projective and $f_{0}$ is surjective, the map $f_{0}$ splits so that Ker $f_{0}$ is a direct summand of $A \otimes_{k G} C_{0}$ as a right $A$-module. Therefore $\operatorname{Ker} f_{0}=\operatorname{Im} f_{1}$ is right $A$-projective. Repeat the argument with $A \otimes_{k G} C_{0}$ replaced by $A \otimes_{k G} C_{1}$ and $A$ replaced by $\operatorname{Im} f_{1}$, and so on, to complete the check.

The Künneth Theorem [29, Theorem 3.6.3] then gives for each $n$ an exact sequence:

$$
\begin{aligned}
0 \longrightarrow \bigoplus_{i+j=n} \mathrm{H}_{i}\left(A \otimes_{k G} C_{\bullet}\right) \otimes_{A} & \mathrm{H}_{j}\left(D_{\bullet} \otimes_{S} A\right) \longrightarrow \mathrm{H}_{n}\left(\left(A \otimes_{k G} C_{\bullet}\right) \otimes_{A}\left(D_{\bullet} \otimes_{S} A\right)\right) \\
& \longrightarrow \bigoplus_{i+j=n-1} \operatorname{Tor}_{1}^{A}\left(\mathrm{H}_{i}\left(A \otimes_{k G} C_{\bullet}\right), \mathrm{H}_{j}\left(D_{\bullet} \otimes_{S} A\right)\right) \rightarrow 0 .
\end{aligned}
$$


Now $A \otimes_{k G} C_{\bullet}$ and $D_{\bullet} \otimes_{S} A$ are exact other than in degree 0 , where their homologies are each $A$. Thus $\mathrm{H}_{j}\left(A \otimes_{k G} C_{\bullet}\right)=\mathrm{H}_{i}\left(D_{\bullet} \otimes_{S} A\right)=0$ unless $i=j=0$. The Tor term for $i=j=0$ is also zero as $\operatorname{Tor}_{1}^{A}(A, A)=0$ (since $A$ is flat over $A$ ). Thus

$$
\mathrm{H}_{n}\left(\left(A \otimes_{k G} C_{\bullet}\right) \otimes_{A}\left(D_{\bullet} \otimes_{S} A\right)\right)=0 \text { for all } n>0
$$

and

$$
\mathrm{H}_{0}\left(\left(A \otimes_{k G} C \bullet\right) \otimes_{A}\left(D \bullet \otimes_{S} A\right)\right) \cong \mathrm{H}_{0}\left(A \otimes_{k G} C \bullet\right) \otimes_{A} \mathrm{H}_{0}\left(D \bullet \otimes_{S} A\right) \cong A \otimes_{A} A \cong A .
$$

Thus $X_{\bullet}$ is an $A^{e}$-free resolution of $A$.

The resolution $X_{\bullet}$, being more general than the one given in [15, §4], has an advantage: One may use any convenient resolution of the group algebra $k G$ in the construction. The resolution in [15] uses the (normalized) bar resolution of $k G$, resulting in a potentially larger complex $X_{\text {• }}$. In the example below, we show that $X$. may be quite tractable when a smaller resolution of $k G$ is chosen.

Example 4.6. Let $G$ be a cyclic group of prime order $p$, generated by $g$. Let $k$ be a field of characteristic $p$. Let $V=k^{2}$ with basis $v_{1}, v_{2}$. Let $g$ act as the matrix

$$
\left(\begin{array}{ll}
1 & 1 \\
0 & 1
\end{array}\right)
$$

on the ordered basis $v_{1}, v_{2}$. Let $S=S_{k}(V)$, the symmetric algebra, and let

$$
D_{\bullet}: \quad 0 \rightarrow S \otimes \bigwedge^{2} V \otimes S \rightarrow S \otimes \bigwedge^{1} V \otimes S \rightarrow S \otimes S \rightarrow S \rightarrow 0
$$

be the Koszul resolution of $S$, where we identify $\bigwedge^{1} V$ with $V$ and $\bigwedge^{2} V$ with $R=\{v \otimes w-w \otimes v \mid v, w \in V\}$. The differentials commute with the $G$-action. Let

$$
C \bullet: \quad \cdots \stackrel{\eta \cdot}{\longrightarrow} k G \otimes k G \stackrel{\gamma \cdot}{\longrightarrow} k G \otimes k G \stackrel{\eta \cdot}{\longrightarrow} k G \otimes k G \stackrel{\gamma \cdot}{\longrightarrow} k G \otimes k G \stackrel{m}{\longrightarrow} k G \rightarrow 0,
$$

where $\gamma=g \otimes 1-1 \otimes g, \eta=g^{p-1} \otimes 1+g^{p-2} \otimes g+\cdots+1 \otimes g^{p-1}$, and $m$ is multiplication. Then $C$ • is a $(k G)^{e}$-free resolution of $k G$; exactness may be verified by constructing an explicit contracting homotopy. We consider $k G \otimes k G$ in even degrees to be a Yetter-Drinfeld module in the usual way: $(k G \otimes k G)_{g^{i}}=\operatorname{Span}_{k}\left\{x \otimes y \mid x y=g^{i}\right\}$. But in odd degrees let $(k G \otimes k G)_{g^{i}}=\operatorname{Span}_{k}\left\{x \otimes y \mid x y=g^{i-1}\right\}$. This will ensure that the differentials preserve the $G$-grading.

By Theorem 4.3, $X_{\bullet \bullet \bullet}=\left(A \otimes_{k G} C_{\bullet}\right) \otimes_{A}\left(D_{\bullet} \otimes_{S} A\right)$ yields a free $A^{e}$-resolution $X_{\bullet}$ (the total complex) of $A=S \# G$. By our earlier analysis (see (4.4)), for all $i \geq 0$ and $0 \leq j \leq 2$,

$$
X_{i, j}=\left(A \otimes_{k G}(k G \otimes k G)\right) \otimes_{A}\left(\left(S \otimes \bigwedge^{j} V \otimes S\right) \otimes_{S} A\right) \cong A \otimes \bigwedge^{j} V \otimes A,
$$

and the differentials are $d=d_{i, j}^{h}+d_{i, j}^{v}=d_{i} \otimes \mathrm{id}+(-1)^{i}$ id $\otimes d_{j}$. Under the given isomorphism, we find that when $i \neq 0$ and $j=0$, for $r, s$ in $S$,

$$
\begin{aligned}
(i \text { odd }) d\left(r g^{a} \otimes s g^{b}\right) & =r g^{a+1} \otimes s g^{b}-r g^{a} \otimes{ }^{g} s g^{b+1}, \quad \text { or } \\
(i \text { even }) d\left(r g^{a} \otimes s g^{b}\right) & =\sum_{c=0}^{p-1} r g^{a+c} \otimes\left(g^{p-1-c} s\right) g^{b+p-1-c} .
\end{aligned}
$$


When $j=1$, if $u \in V$,

$$
\begin{aligned}
(i \text { odd }) d\left(r g^{a} \otimes u \otimes s g^{b}\right)= & -\left(r g^{a} u \otimes s g^{b}-r g^{a} \otimes u s g^{b}\right) \\
& +\left(r g^{a+1} \otimes u \otimes s g^{b}-r g^{a} \otimes{ }^{g} u \otimes{ }^{g} s g^{b+1}\right), \quad \text { or } \\
(i \text { even }) d\left(r g^{a} \otimes u \otimes s g^{b}\right)= & \left(r g^{a} u \otimes s g^{b}-r g^{a} \otimes u s g^{b}\right) \\
& +\sum_{c=0}^{p-1} r g^{a+c} \otimes g^{p-1-c} u \otimes\left(g^{p-1-c} s\right) g^{b+p-1-c},
\end{aligned}
$$

unless $i=0$, in which case the sum over $c$ should be deleted since the corresponding differential is 0 in that case. When $j=2$,

$$
\begin{aligned}
& (i \text { odd }) d\left(r g^{a} \otimes v_{1} \wedge v_{2} \otimes s g^{b}\right)=-\left(r g^{a} v_{1} \otimes v_{2} \otimes s g^{b}-r g^{a} \otimes v_{2} \otimes v_{1} s g^{b}\right. \\
& \left.-r g^{a} v_{2} \otimes v_{1} \otimes s g^{b}+r g^{a} \otimes v_{1} \otimes v_{2} s g^{b}\right) \\
& +r g^{a+1} \otimes v_{1} \wedge v_{2} \otimes s g^{b} \\
& -r g^{a} \otimes v_{1} \wedge v_{2} \otimes{ }^{g} s g^{b+1}, \quad \text { or } \\
& (i \text { even }) d\left(r g^{a} \otimes v_{1} \wedge v_{2} \otimes s g^{b}\right)=\left(r g^{a} v_{1} \otimes v_{2} \otimes s g^{b}-r g^{a} \otimes v_{2} \otimes v_{1} s g^{b}\right. \\
& \left.-r g^{a} v_{2} \otimes v_{1} \otimes s g^{b}+r g^{a} \otimes v_{1} \otimes v_{2} s g^{b}\right) \\
& +\sum_{c=0}^{p-1} r g^{a+c} \otimes v_{1} \wedge v_{2} \otimes\left(g^{p-1-c} s\right) g^{b+p-1-c},
\end{aligned}
$$

unless $i=0$, in which case the sum over $c$ should be deleted.

For our applications to Koszul algebras, we will need chain maps between the resolution $X_{\bullet}$ and the bar resolution of $A$ with the properties stated in the next lemma.

Lemma 4.7. Let $S$ be a finitely generated graded Koszul algebra over $k$ on which a finite group $G$ acts by graded automorphisms. Let $C$. be the bar resolution of $k G$, let $D_{\bullet}$ be the Koszul resolution of $S$, let $A=S \# G$, and let $X_{\bullet}$ be as in (4.2). Then there exist chain maps $\phi_{\bullet}: X_{\bullet} \rightarrow A^{\otimes(\bullet+2)}$ and $\psi_{\bullet}: A^{\otimes(\bullet+2)} \rightarrow X_{\bullet}$ of degree 0 for which $\psi_{n} \phi_{n}$ is the identity map on the subspace $X_{0, n}$ of $X_{n}$ for each $n \geq 0$.

A chain map was given explicitly in case $S=S_{k}(V)$ by Guccione, Guccione, and Valqui [15, §4.2].

Proof. Both $X_{\bullet}$ and $A^{\otimes(\bullet+2)}$ are free resolutions of $A$ as an $A^{e}$-module whose differentials are maps of degree 0 . We first argue inductively that there exists a chain map $\phi_{n}: X_{n} \rightarrow A^{\otimes(n+2)}$ of degree 0 for which $\left.\phi_{n}\right|_{X_{0, n}}$ is induced by the standard embedding of the Koszul complex into the bar complex.

Suppose $S$ is generated by a finite dimensional $k$-vector space $V$ with quadratic relations $R: S=T_{k}(V) /\langle R\rangle$ (see Section 3). Define $\phi_{0}=\mathrm{id} \otimes \mathrm{id}=\psi_{0}$, the identity map from $A \otimes A$ to itself. Consider $X_{0}$, as a subcomplex (not necessarily acyclic) of $X_{\bullet}$. An inductive argument shows that we may define $\phi_{\bullet}$ so that when restricted to $X_{0}$, it corresponds to the standard embedding of the Koszul complex into the bar complex: For $n=1$, this is the embedding of $A \otimes V \otimes A$ into $A \otimes A \otimes A$, and one checks that $\phi_{1}$ on $X_{1}=X_{0,1} \oplus X_{1,0} \cong(A \otimes V \otimes A) \oplus(A \otimes k G \otimes A)$ may be defined by $\phi_{1}(1 \otimes v \otimes 1)=1 \otimes v \otimes 1$ and $\phi_{1}(1 \otimes g \otimes 1)=1 \otimes g \otimes 1$ for all $v \in V$, 
$g \in G$. For $n \geq 2$,

$$
X_{0, n} \cong A \otimes\left(\bigcap_{i=0}^{n-2} V^{\otimes i} \otimes R \otimes V^{\otimes(n-i-2)}\right) \otimes A,
$$

a free $A^{e}$-submodule of $A^{\otimes(n+2)}$ by its definition. For each $i, j$ with $i+j=n$, choose a basis of the vector space $C_{i}^{\prime} \otimes D_{j}^{\prime}$, whose elements are necessarily of degree $j$. By construction, after applying $\phi_{n-1} d_{n}$ to these basis elements, we obtain elements of degree $j$ in the kernel of $\delta_{n-1}$, which is the image of $\delta_{n}$. Choose corresponding elements in the inverse image of $\operatorname{Im}\left(\delta_{n}\right)$ to define $\phi_{n}$. If we start with an element in $X_{0, n}$, we may choose its canonical image in $A^{\otimes(n+2)}$ (see (4.8)). Elements of $X_{i, j}$ $(i>0)$ have different degree, so their images under $\phi_{n}$ may be chosen independently of those of $X_{0, n}$.

Now we show inductively that each $\psi_{n}$ may be chosen to be a degree 0 map for which $\psi_{n} \phi_{n}$ is the identity map on $X_{0, n}$. In degree 0 , this is true as $\phi_{0}, \psi_{0}$ are identity maps. In degree $1, X_{0,1} \cong A \otimes V \otimes A$ and $X_{1,0} \cong A \otimes k G \otimes A$. Note that $V \oplus k G$ is a direct summand of $A$ as a vector space. We may therefore define $\psi_{1}(1 \otimes v \otimes 1)=1 \otimes v \otimes 1$ in $X_{0,1}$ for all $v \in V$ and $\psi_{1}(1 \otimes g \otimes 1)=1 \otimes g \otimes 1$ in $X_{1,0}$ for all $g \in G$. We define $\psi_{1}$ on elements of the form $1 \otimes z \otimes 1$, for $z$ ranging over a basis of a chosen complement of $V \oplus k G$ as a vector subspace of $A$, arbitrarily subject to the condition that $d_{1} \psi_{1}(1 \otimes z \otimes 1)=\psi_{0} \delta_{1}(1 \otimes z \otimes 1)$. Since $\psi_{0}, d_{1}, \delta_{1}$ all have degree 0 as maps, one may also choose $\psi_{1}$ to have degree 0 . Note that $\psi_{1} \phi_{1}$ is the identity map on $X_{0,1}$. (In fact, it is the identity map on all of $X_{1}$.) Now let $n \geq 2$ and assume that $\psi_{n-2}$ and $\psi_{n-1}$ have been defined to be degree 0 maps for which $d_{n-1} \psi_{n-1}=\psi_{n-2} \delta_{n-1}$ and $\psi_{j} \phi_{j}$ is the identity map on $X_{0, j}$ for $j=n-2, n-1$. To define $\psi_{n}$, first note that $A^{\otimes(n+2)}$ contains as an $A^{e_{-}}$ submodule the space $X_{0, n}$ (see (4.8)) and the image of each $X_{i, j}$ under $\phi_{n}(n=i+j$, $i \geq 1$ ). By construction, their images intersect in 0 (being generated by elements of different degrees), the image of $X_{0, n}$ under $\phi_{n}$ is free, and moreover $\phi_{n}$ is injective on restriction to $X_{0, n}$. Choose a set of free generators of $\phi_{n}\left(X_{0, n}\right)$, and choose a set of free generators of its complement in $A^{\otimes(n+2)}$. For each chosen generator $x$ of $X_{0, n}$, we define $\psi_{n}\left(\phi_{n}(x)\right)$ to be $x$. Since $d_{n}(x)$ is in $X_{0, n-1}$ by definition, we have by induction $\psi_{n-1} \phi_{n-1} d_{n}(x)=d_{n}(x)$. As $\delta_{n} \phi_{n}(x)=\phi_{n-1} d_{n}(x)$, we now have $d_{n} \psi_{n} \phi_{n}(x)=\psi_{n-1} \delta_{n} \phi_{n}(x)$. That is, on these elements, $\psi_{n}$ extends the chain map from degree $n-1$ to degree $n$. On the remaining free generators of $A^{\otimes(n+2)}$, define $\psi_{n}$ arbitrarily subject to the requirement that it be a chain map of degree 0 .

\section{Deformations of QUadratic algebras}

Let $B$ be an arbitrary $k$-algebra. Let $U$ be a $B$-bimodule that is free as a left $B$-module and as a right $B$-module, and set

$$
T:=T_{B}(U)=B \oplus U \oplus\left(U \otimes_{B} U\right) \oplus\left(U \otimes_{B} U \otimes_{B} U\right) \oplus \cdots,
$$

the tensor algebra of $U$ over $B$ with the $i$-th graded component $T^{i}:=U^{\otimes_{B}}{ }^{i}$ and $T^{0}=B$. Let $F^{i}(T)$ be the $i$-th filtered component: $F^{i}(T)=T^{0} \oplus T^{1} \oplus \cdots \oplus T^{i}$. We call a $B$-subbimodule $P$ of $F^{2}(T)$ a set of filtered quadratic relations over $B$, and we call the quotient $T_{B}(U) /\langle P\rangle$ a filtered quadratic algebra over $B$ generated by $U$. By Proposition 2.1, if the relations are of PBW type, then they are unique. Set $R=\pi(P)$ where (recall) $\pi$ is the projection $F^{2}(T) \rightarrow U \otimes_{B} U$, so 
that $T /\langle R\rangle$ is the homogeneous quadratic algebra determined by $P$. Note that $R$ is a $B$-subbimodule of $U \otimes_{B} U$.

We give below some conditions sufficient to guarantee that $P$ and the quadratic algebra $T_{B}(U) /\langle P\rangle$ it defines are of PBW type. First we present two lemmas. It is not difficult to see that any quadratic algebra over $B$ of PBW type must be defined by a $B$-subbimodule $P \subset T$ devoid of elements of filtered degree one. We record this observation and more in the next lemma. We choose labels consistent with those in 5 ] for ease of comparison. The proof (see (2.2) ) of Proposition 2.1]implies:

Lemma 5.1. Suppose $P \subset T$ is a set of filtered quadratic relations over $B$ defining a filtered quadratic algebra $T /\langle P\rangle$ of $P B W$ type (with respect to $P$ ). Then

(I) $P \cap F^{1}(T)=\{0\}$, and

(J) $\quad\left(F^{1}(T) P F^{1}(T)\right) \cap F^{2}(T)=P$.

If condition (I) of Lemma 5.1 holds, then each (nonhomogeneous) generating relation defining the quadratic algebra $T /\langle P\rangle$ may be expressed as a unique element of homogeneous degree 2 plus linear and constant terms. We record these terms with functions $\alpha$ and $\beta$ : Condition (I) implies the existence of $k$-linear maps $\alpha: R \rightarrow U$ and $\beta: R \rightarrow B$ for which

$$
P=\{x-\alpha(x)-\beta(x) \mid x \in R\} .
$$

Since $P$ is a $B$-subbimodule of $T$, so is $R$, and it is not hard to see that the maps $\alpha$ and $\beta$ are $B$-bimodule homomorphisms. We may now use the maps $\alpha$ and $\beta$ to explore the PBW property using cohomology instead of examining overlap polynomials and ambiguities (see [6], for example) explicitly. Note that since $U$ is free (and thus flat) as a left $B$-module and as a right $B$-module, the spaces $R \otimes_{B} U$ and $U \otimes_{B} R$ may be identified with subspaces of $U^{\otimes_{B} 3}$.

Lemma 5.2. Suppose $P \subset T$ is a set of filtered quadratic relations over $B$ defining a filtered quadratic algebra $T /\langle P\rangle$ of $P B W$ type (with respect to $P$ ). Then

(i) $\operatorname{Im}\left(\alpha \otimes_{B}\right.$ id - id $\left.\otimes_{B} \alpha\right) \subset R$,

(ii) $\alpha \circ\left(\alpha \otimes_{B}\right.$ id - id $\left.\otimes_{B} \alpha\right)=-\left(\beta \otimes_{B}\right.$ id - id $\left.\otimes_{B} \beta\right)$,

(iii) $\beta \circ\left(\alpha \otimes_{B}\right.$ id - id $\left.\otimes_{B} \alpha\right)=0$,

where the maps $\alpha \otimes_{B} \mathrm{id}-\mathrm{id} \otimes_{B} \alpha$ and $\beta \otimes_{B} \mathrm{id}-\mathrm{id} \otimes_{B} \beta$ are defined on the subspace $\left(R \otimes_{B} U\right) \cap\left(U \otimes_{B} R\right)$ of $T$.

Proof. By Lemma 5.1. conditions (I) and (J) hold. We show that (I) and (J) imply (i), (ii), and (iii). Let $x \in\left(R \otimes_{B} U\right) \cap\left(U \otimes_{B} R\right)$. By the definitions of $\alpha$ and $\beta$,

$$
\begin{aligned}
& x-\left(\alpha \otimes_{B} \text { id }+\beta \otimes_{B} \text { id }\right)(x) \in P T^{1} \subset F^{1}(T) P F^{1}(T), \\
& x-\left(\text { id } \otimes_{B} \alpha+\mathrm{id} \otimes_{B} \beta\right)(x) \in T^{1} P \subset F^{1}(T) P F^{1}(T) .
\end{aligned}
$$

We subtract these two expressions and check degrees to see that condition $(\mathrm{J})$ implies

$\left(\alpha \otimes_{B}\right.$ id - id $\otimes_{B} \alpha+\beta \otimes_{B}$ id - id $\left.\otimes_{B} \beta\right)(x) \in\left(F^{1}(T) P F^{1}(T)\right) \cap F^{2}(T)=P$.

Again considering the degrees of the above elements, we must have

$$
\begin{aligned}
\left(\alpha \otimes_{B} \text { id }- \text { id } \otimes_{B} \alpha\right)(x) & \in \\
\alpha\left(\left(\alpha \otimes_{B} \text { id }- \text { id } \otimes_{B} \alpha\right)(x)\right) & =-\left(\beta \otimes_{B} \text { id }- \text { id } \otimes_{B} \beta\right)(x), \\
\beta\left(\left(\alpha \otimes_{B} \text { id }- \text { id } \otimes_{B} \alpha\right)(x)\right) & =0 .
\end{aligned}
$$


Example 5.3. We return to Example 2.3 in which $B=k$ and the $B$-bimodule $U$ is the vector space $V=\operatorname{Span}_{k}\{x, y\}$ with $P^{\prime}=\operatorname{Span}_{k}\left\{x y-x, y x-y, x^{2}-x, y^{2}-y\right\}$. As $\beta$ is identically zero, an easy check of conditions (i), (ii), and (iii) amounts to checking overlap relations in $P^{\prime}$ and verifying that $P^{\prime}$ is a noncommutative Gröbner basis for the ideal it generates in $T_{k}(V)$.

In the remainder of this section, we turn to the case $B=k G$, a finite group algebra. We show in the next theorem that the above conditions, adapted from Braverman and Gaitsgory [5, are both necessary and sufficient in the case that the homogeneous quadratic algebra determined by $P$ is isomorphic to $S \# G$ for some Koszul algebra $S$. (Precisely, we set $U=V \otimes k G$ for a finite dimensional $k$-vector space $V$ and view $U$ as a bimodule over the group algebra $B=k G$.)

Theorem 5.4. Let $S$ be a finitely generated graded Koszul algebra over $k$ on which a finite group $G$ acts by graded automorphisms. Suppose a filtered quadratic algebra $A^{\prime}$ over $k G$ is defined by a set of filtered quadratic relations that determine a homogeneous quadratic algebra isomorphic to $S \# G$. Then $A^{\prime}$ is of $P B W$ type if and only if conditions (I), (i), (ii), and (iii) hold.

Proof. Suppose the Koszul algebra $S$ is generated by the $k$-vector space $V$ with some $k$-vector space of quadratic relations $R^{\prime} \subset V \otimes V$, i.e., $S=T_{k}(V) /\left\langle R^{\prime}\right\rangle$. Let $U=V \otimes k G$, a $k G$-bimodule with right action given by multiplication on the rightmost factor $k G$ only and left action given by ${ }^{g}(v \otimes h)={ }^{g} v \otimes g h$ for all $v \in V$, $g, h \in G$. Set $R=R^{\prime} \otimes k G$, similarly a $k G$-bimodule (as $R^{\prime}$ is a $k G$-module). Then

$$
T_{k G}(U) /\langle R\rangle \cong\left(T_{k}(V) /\left\langle R^{\prime}\right\rangle\right) \# G=S \# G \cong\left(T_{k}(V) \# G\right) /\left\langle R^{\prime}\right\rangle
$$

as a consequence of the canonical isomorphism between $T_{k G}(U)$ and $T_{k}(V) \# G$ given by identifying elements of $V$ and of $G$ and moving all group elements far right; here $\left\langle R^{\prime}\right\rangle$ denotes the ideal of $T_{k}(V)$ or of $T_{k}(V) \# G$ generated by $R^{\prime}$, respectively.

We may assume that $A^{\prime}$ is also generated by $U$ over $k G$, i.e., $A^{\prime}=T_{k G}(U) /\langle P\rangle$ for a set of filtered quadratic relations $P$ over $k G$. Note that $\pi(P)=R$ since both $\pi(P)$ and $R$ are $k G$-bimodules generating the same ideal in $T_{k G}(U)$ (use Proposition 2.1, for example). The conditions are then necessary by Lemmas 5.1 and 5.2 . It remains to prove that they are sufficient, so assume conditions (I), (i), (ii), and (iii) hold for $P=\{x-\alpha(x)-\beta(x) \mid x \in R\}$.

We adapt the proof of Braverman and Gaitsgory [5, §4] to our setting using the resolution $X_{\bullet}$ given by (4.2) after choosing $C \bullet$ to be the bar resolution (3.1) of $k G$ and $D_{\bullet}$ to be the Koszul resolution (3.7) of $S$. By Theorem 4.3, $X_{\bullet}$ calculates the Hochschild cohomology $\mathrm{HH}^{\bullet}(A)$ cataloging deformations of $A$. We first extend the maps $\alpha$ and $\beta$ to cochains on $X_{\bullet}$. We then use chain maps between $X_{\bullet}$ and the bar resolution for $A$ to convert $\alpha$ and $\beta$ to Hochschild 2-cochains which can define multiplication maps for a potential deformation. We modify the cochains as necessary to preserve the conditions of the theorem. Using these conditions, we build a second level graded deformation of $A$. We then extend to a graded deformation of $A$ and conclude the PBW property using properties of the resolution $X_{\bullet}$. We note that conditions (i), (ii), and (iii) may be interpreted as conditions on a tensor product over $k$ for the extensions of the maps $\alpha, \beta$ to $X_{\bullet}$.

We first extend $\alpha$ and $\beta$ to $X_{\bullet}$. In degree $2, X_{2}$ contains the direct summand $X_{0,2} \cong A \otimes R^{\prime} \otimes A$ (apply Theorem 4.3 with $i=0, j=2$ ). Note that $R=R^{\prime} \otimes k G \subseteq$ $V \otimes V \otimes k G \cong U \otimes_{k G} U$, and we thus view the $k G$-bilinear maps $\alpha, \beta: R \rightarrow A$ as 
maps on $R^{\prime} \otimes k G$. Extend them to $A^{e}$-module maps from $A \otimes R^{\prime} \otimes A \cong A \otimes R \otimes S$ to $A$ by composing with the multiplication map, and, by abuse of notation, denote these extended maps by $\alpha, \beta$ as well. Set $\alpha$ and $\beta$ equal to 0 on the summands $X_{2,0}$ and $X_{1,1}$ of $X_{2}$ so that they further extend to maps $\alpha, \beta: X_{2} \rightarrow A$.

Condition (i) implies that $\alpha$ is 0 on the image of the differential on $X_{0,3}$. Since $\alpha$ is a $k G$-bimodule homomorphism by its definition, $\alpha$ is $G$-invariant. We claim that this implies it is also 0 on the image of the differential on $X_{1,2}$ : Let $a, b \in A, g \in G$, and $r \in R^{\prime}$, and consider $a \otimes g \otimes r \otimes b$ as an element of $X_{1,2} \cong A \otimes k G \otimes R^{\prime} \otimes A$ (apply Theorem 4.3 with $i=1, j=2$ ). We apply (4.5):

$$
\begin{aligned}
d(a \otimes g \otimes r \otimes b) & =d((a \otimes g \otimes 1) \otimes(1 \otimes r \otimes b)) \\
& =d(a \otimes g \otimes 1) \otimes(1 \otimes r \otimes b)-(a \otimes g \otimes 1) \otimes d(1 \otimes r \otimes b) .
\end{aligned}
$$

The second term lies in $X_{1,1}$, but $\alpha$ is 0 on $X_{1,1}$ by definition. Therefore

$$
\begin{aligned}
\alpha(d(a \otimes g \otimes r \otimes b)) & =\alpha((a g \otimes 1-a \otimes g) \otimes(1 \otimes r \otimes b)) \\
& =\alpha\left(a g \otimes r \otimes b-a \otimes{ }^{g} r \otimes g b\right) \\
& =a g \alpha(r) b-a \alpha\left({ }^{g} r\right) g b,
\end{aligned}
$$

where we use (4.4). But $g \alpha(r)=\alpha\left({ }^{g} r\right) g$, since $\alpha$ is $G$-invariant, and thus $\alpha$ is zero on the image of $d$ on $X_{1,2}$. It follows that $\alpha$ is a 2-cocycle on $X_{\bullet}$ and defines a Hochschild cohomology class of $\operatorname{HH}^{2}(A)$. Thus $\alpha$ yields a first level deformation $A_{1}$ of $A$ (i.e., an infinitesimal deformation of $A$ ) with some first multiplication map $\mu_{1}: A \otimes A \rightarrow A$.

In fact, we may apply the chain map $\psi_{\bullet}$ of Lemma 4.7 and choose $\mu_{1}=\psi_{2}^{*}(\alpha)$. Note that $\alpha$ is homogeneous of degree -1 by its definition, and therefore so is $\mu_{1}$. We claim that $\phi_{2}^{*}\left(\mu_{1}\right)=\alpha$ as cochains. To verify this, first let $x \in X_{0,2}$. By Lemma 4.7. $\psi_{2} \phi_{2}(x)=x$, and hence

$$
\mu_{1} \phi_{2}(x)=\alpha \psi_{2} \phi_{2}(x)=\alpha(x) .
$$

Now let $x$ be a free generator of $X_{1,1}$ or of $X_{2,0}$, so that it has degree 1 or 0 . Then $\psi_{2} \phi_{2}(x)$ has degree 1 or 0 , implying that its component in $X_{0,2}$ is 0 , from which it follows that $\mu_{1} \phi_{2}(x)=\alpha \psi_{2} \phi_{2}(x)=0=\alpha(x)$. Therefore $\phi_{2}^{*}\left(\mu_{1}\right)=\alpha$.

Condition (ii) implies that $-d_{3}^{*}(\beta)=\alpha \circ(\alpha \otimes$ id - id $\otimes \alpha)$ as cochains on $X$. (Again, since $\beta$ is $G$-invariant, it will be 0 on the image of the differential on $X_{1,2}$.) Let $\mu_{2}=\psi_{2}^{*}(\beta)$. By a similar argument as that above for $\alpha$, we have $\phi_{2}^{*}\left(\mu_{2}\right)=\beta$. However, we want $\mu_{1}, \mu_{2}$ to satisfy the differential condition that $\alpha, \beta$ satisfy, i.e.,

$$
-\delta^{*}\left(\mu_{2}\right)=\mu_{1} \circ\left(\mu_{1} \otimes \mathrm{id}-\mathrm{id} \otimes \mu_{1}\right)
$$

as cochains on the bar resolution. We modify $\mu_{2}$ as necessary to satisfy this condition. Let

$$
\gamma=\delta^{*}\left(\mu_{2}\right)+\mu_{1} \circ\left(\mu_{1} \otimes \mathrm{id}-\mathrm{id} \otimes \mu_{1}\right) .
$$

The cochain $\phi^{*}(\gamma)$ is zero on $X_{0,3}$ by condition (ii), since the image of $\phi$ on $X_{0,3}$ is contained in $A \otimes\left(\left(R^{\prime} \otimes V\right) \cap\left(V \otimes R^{\prime}\right)\right) \otimes A$, and $\phi^{*}\left(\mu_{1}\right)=\alpha$. Additionally, $\phi^{*}(\gamma)$ is 0 on $X_{2,1}$ and on $X_{3,0}$ since it is a map of degree -2 . To see that it is also 0 on $X_{1,2}$, note that as an $A^{e}$-module, the image of $X_{1,2}$ under $\phi$ is generated by elements of degree 2 . Since $\alpha$ contains $k G$ in its kernel and $\mu_{1}=\psi_{2}^{*}(\alpha)$, the map $\mu_{1} \circ\left(\mu_{1} \otimes \mathrm{id}-\mathrm{id} \otimes \mu_{1}\right)$ must be 0 on the image of $X_{1,2}$ under $\phi$. Since $\beta$ is $G$-invariant and $\phi^{*}\left(\mu_{2}\right)=\beta$, we have that $\phi^{*} \delta^{*}\left(\mu_{2}\right)=d^{*} \phi^{*}\left(\mu_{2}\right)=d^{*}(\beta)$ is 0 on $X_{1,2}$. Therefore $\phi^{*}(\gamma)$ is 0 on $X_{1,2}$. We have shown that $\phi^{*}(\gamma)$ is 0 on all of $X_{3}$, and so $\gamma$ defines the zero cohomology class on the bar complex as well, i.e., 
it is a coboundary. Thus there is a 2-cochain $\mu$ of degree -2 on the bar complex with $\delta^{*}(\mu)=\gamma$. If we were to replace $\mu_{2}$ with $\mu_{2}-\mu$, it would satisfy the desired differential condition (5.5) . However, $\phi^{*}\left(\mu_{2}-\mu\right)$ may not agree with $\beta$ on $X_{2}$. We subtract off another term: Since

$$
d^{*} \phi^{*}(\mu)=\phi^{*} \delta^{*}(\mu)=\phi^{*}(\gamma)=0,
$$

the 2-cochain $\phi^{*}(\mu)$ is a cocycle on the complex $X_{\bullet}$ and thus lifts to a cocycle $\mu^{\prime}$ of degree -2 on the bar complex, i.e., $\mu^{\prime}$ satisfies $\phi^{*}\left(\mu^{\prime}\right)=\phi^{*}(\mu)$. Then

$$
\phi^{*}\left(\mu_{2}-\mu+\mu^{\prime}\right)=\beta
$$

and

$$
\delta^{*}\left(\mu_{2}-\mu+\mu^{\prime}\right)+\mu_{1} \circ\left(\mu_{1} \otimes \mathrm{id}-\mathrm{id} \otimes \mu_{1}\right)
$$

is zero on the bar resolution as $\delta^{*} \mu^{\prime}=0$ and $-\delta^{*}\left(\mu_{2}-\mu\right)=\mu_{1} \circ\left(\mu_{1} \otimes \mathrm{id}-\mathrm{id} \otimes \mu_{1}\right)$. We hence replace $\mu_{2}$ by $\mu_{2}-\mu+\mu^{\prime}$.

We have now constructed maps $\mu_{1}, \mu_{2}$ satisfying the differential condition (5.5) required to obtain a second level graded deformation: There exists a second level graded deformation $A_{2}$ of $A$ (with multiplication defined by $\mu_{2}$ ) extending $A_{1}$.

By condition (iii) and degree considerations, the obstruction

$$
\mu_{2} \circ\left(\mu_{1} \otimes \text { id }- \text { id } \otimes \mu_{1}\right)+\mu_{1} \circ\left(\mu_{2} \otimes \text { id }- \text { id } \otimes \mu_{2}\right)
$$

to lifting $A_{2}$ to a third level deformation $A_{3}$ is 0 as a cochain on $X_{\bullet}$ under the cochain map $\phi^{*}$. Therefore, as a cochain on the bar resolution, this obstruction is a coboundary, and so represents the zero cohomology class in $\mathrm{HH}^{3}(A)$. Thus there exists a 2-cochain $\mu_{3}$ of degree -3 satisfying the obstruction equation (3.5) for $i=3$, and the deformation lifts to the third level by Proposition 3.6 .

The obstruction for a third level graded deformation $A_{3}$ of $A$ to lift to the fourth level lies in $\mathrm{HH}^{3,-4}(A)$ (again by Proposition 3.6). We apply $\phi^{*}$ to this obstruction (the right side of equation (3.5) with $i=4$ ) to obtain a cochain on $X_{3}$. But there are no cochains of degree -4 on $X_{3}$ by definition (as it is generated by elements of degree 3 or less), hence the obstruction is automatically zero. Therefore the deformation may be continued to the fourth level. Similar arguments show that it can be continued to the fifth level, and so on.

Let $A_{t}$ be the (graded) deformation of $A$ that we obtain in this manner. Then $A_{t}$ is the $k$-vector space $A[t]$ with multiplication

$$
a_{1} * a_{2}=a_{1} a_{2}+\mu_{1}\left(a_{1} \otimes a_{2}\right) t+\mu_{2}\left(a_{1} \otimes a_{2}\right) t^{2}+\mu_{3}\left(a_{1} \otimes a_{2}\right) t^{3}+\ldots,
$$

where $a_{1} a_{2}$ is the product in the homogeneous quadratic algebra $A=S \# G \cong$ $T_{k G}(U) /\langle R\rangle \cong\left(T_{k}(V) \# G\right) /\left\langle R^{\prime}\right\rangle$ and each $\mu_{i}: A \otimes A \rightarrow A$ is a $k$-linear map of homogeneous degree $-i$. (The sum terminates for each pair $a_{1}, a_{2}$ by degree considerations.) Then for any $r$ in $R, \mu_{1}(r)=\alpha(r)$ and $\mu_{2}(r)=\beta(r)$ by construction, and $\mu_{i}(r)=0$ for $i \geq 3$ by considering degrees.

We now argue that $A^{\prime}$ is isomorphic as a filtered algebra to the fiber of the deformation $A_{t}$ at $t=1$. Let $A^{\prime \prime}=\left.\left(A_{t}\right)\right|_{t=1}$. First note that $A^{\prime \prime}$ is generated by $V$ and $G$, since it is a filtered algebra (as $A_{t}$ is graded) with associated graded algebra $A=S \# G$. Next note that products of pairs of elements in $G$, or of an element of $V$ paired with an element of $G$, are the same in $A^{\prime \prime}$ as they are in $T_{k}(V) \# G$ : $g * v=g v, v * g=v g$, and $g * h=g h$ for all $g, h$ in $G$. To verify this observation, one needs only to check that $\mu_{1}$ vanishes on such pairs, as $A_{t}$ is a graded deformation with group elements in degree 0 and vectors in degree 1 . But $\mu_{1}$ must vanish on 
low degree pairs by our construction of chain maps: $\mu_{1}=\psi_{2}^{*}(\alpha)$, the chain map $\psi_{2}$ preserves degree, and $X_{0,2}$ has free basis as an $A^{e}$-module consisting of elements of degree 2. Thus the canonical surjective algebra homomorphism

$$
T_{k G}(U) \cong T_{k}(V) \# G \rightarrow A^{\prime \prime}
$$

arises, mapping each $v$ in $V$ and $g$ in $G$ to their copies in $A^{\prime \prime}$. The elements of $P$ lie in the kernel (by definition of $A^{\prime \prime}$ ), and thus the map induces a surjective algebra homomorphism:

$$
A^{\prime}=T_{k G}(U) /\langle P\rangle \rightarrow A^{\prime \prime} .
$$

We claim this map is an isomorphism of filtered algebras. First compare the dimensions in each filtered component of these two algebras. Recall that algebra $A^{\prime}$ has dimension at most that of $S \# G$ in each filtered component (as its associated graded algebra is a quotient of $S \# G)$. Then since there is a surjective algebra homomorphism from $A^{\prime}$ to $A^{\prime \prime}$ and $\operatorname{gr}\left(A^{\prime \prime}\right)=S \# G$,

$$
\operatorname{dim}_{k}\left(F^{m}(S \# G)\right) \geq \operatorname{dim}_{k}\left(F^{m}\left(A^{\prime}\right)\right) \geq \operatorname{dim}_{k}\left(F^{m}\left(A^{\prime \prime}\right)\right)=\operatorname{dim}_{k}\left(F^{m}(S \# G)\right)
$$

for each degree $m$, where $F^{m}$ denotes the $m$-th filtered component. Thus the inequalities are forced to be equalities, and $A^{\prime} \cong A^{\prime \prime}$, a specialization of a deformation of $S \# G$. Consequently $A^{\prime}$ is of PBW type.

The next result points out a correspondence between PBW filtered quadratic algebras and fibers of graded deformations of a particular type:

Corollary 5.6. Let $A=S \# G$ for a finitely generated, graded Koszul algebra $S$ over $k$ generated by a vector space $V$ carrying the action of a finite group $G$ by graded automorphisms. Every graded deformation $A_{t}$ of $A$ for which the kernel of $\mu_{1}$ contains $k G \otimes V$ and $V \otimes k G$ has fiber at $t=1$ isomorphic (as a filtered algebra) to a filtered quadratic algebra over $k G$ of $P B W$ type with induced quadratic algebra isomorphic to A. Conversely, every such filtered quadratic algebra is isomorphic to the fiber at $t=1$ of a graded deformation of $A$ for which the kernel of $\mu_{1}$ contains $k G \otimes V$ and $V \otimes k G$.

Proof. Write the algebra $A=S \# G$ as $T_{k G}(U) /\langle R\rangle$, where $U=V \otimes k G$ for some finite dimensional $k$-vector space $V$ generating $S$ and a set of filtered quadratic relations $R \subset U \otimes_{k G} U$. Suppose $A_{t}$ is a graded deformation of $A$ with multiplication map $\mu_{1}$ vanishing on all $v \otimes g$ and $g \otimes v$ for $g$ in $G$ and $v$ in $V$. (Higher degree maps automatically vanish on such input as $A_{t}$ is graded.) We may reverse engineer the filtered quadratic algebra $A^{\prime}=T_{k G}(U) /\langle P\rangle$ by setting $P=\left\{r-\mu_{1}(r)-\mu_{2}(r)\right.$ : $r \in R\}$. The argument at the end of the proof of Theorem 5.4 implies that $A^{\prime}$ is of PBW type and isomorphic to the fiber of $A_{t}$ at $t=1$ as a filtered algebra, as this fiber is a quotient of $T_{k}(V) \# G$. Conversely, any PBW filtered quadratic algebra satisfies the conditions of Theorem 5.4, its proof constructs a graded deformation $A_{t}$, for which the kernel of $\mu_{1}$ contains $k G \otimes V$ and $V \otimes k G$, and whose fiber $A^{\prime \prime}$ at $t=1$ is isomorphic to $A^{\prime}$ as a filtered algebra.

\section{Applications: Drinfeld orbifold algebras, graded Hecke algebras, AND SYMPLECTIC REFLECTION ALGEBRAS}

We now apply our results from previous sections to Drinfeld orbifold algebras, which include graded Hecke algebras, rational Cherednik algebras, symplectic reflection algebras, and Lie orbifold algebras as special cases. These algebras present 
as a certain kind of quotient of a skew group algebra. Let $G$ be a finite group acting linearly on a finite dimensional $k$-vector space $V$, and consider the induced action on $S_{k}(V)$ and on $T_{k}(V)$. Drinfeld orbifold algebras are deformations of the skew group algebra $S_{k}(V) \# G$ (see [27]). Many articles investigate their properties and representation theory, in particular, when $k$ is the field of real or complex numbers, when $G$ acts faithfully, and when $G$ acts symplectically. We are interested in the case when the characteristic of $k$ divides $|G|$, as the theory is much less developed.

Drinfeld orbifold algebras arise as quotients of the form

$$
\mathcal{H}_{\kappa}=T_{k}(V) \# G /\left\langle v_{1} \otimes v_{2}-v_{2} \otimes v_{1}-\kappa\left(v_{1}, v_{2}\right): v_{1}, v_{2} \in V\right\rangle,
$$

where $\kappa$ is a parameter function on $V \otimes V$ taking values in the group algebra $k G$, or possibly in $V$, or some combination of $k G$ and $V$. We abbreviate $v \otimes 1$ by $v$ and $1 \otimes g$ by $g$ in the skew group algebra $T_{k}(V) \# G$ (which is isomorphic to $T_{k}(V) \otimes k G$ as a $k$-vector space). We take $\kappa$ to be any alternating map from $V \otimes V$ to the first filtered component of $T_{k}(V) \# G$ :

$$
\kappa: V \otimes V \rightarrow k G \oplus(V \otimes k G),
$$

and write $\kappa\left(v_{1}, v_{2}\right)$ for $\kappa\left(v_{1} \otimes v_{2}\right)$ for ease of notation. The associated graded algebra of $\mathcal{H}_{\kappa}$ is a quotient of $S_{k}(V) \# G$. We say that $\mathcal{H}_{\kappa}$ is a Drinfeld orbifold algebra if it satisfies the Poincaré-Birkhoff-Witt property: $\operatorname{gr}\left(\mathcal{H}_{\kappa}\right) \cong S_{k}(V) \# G$ as graded algebras. We explained in [27] how every Drinfeld orbifold algebra defines a formal deformation of $S_{k}(V) \# G$ and also explained which deformations arise this way explicitly.

Various authors explore conditions on $\kappa$ guaranteeing that the quotient $\mathcal{H}_{\kappa}$ satisfies the PBW property. Such Drinfeld orbifold algebras are often called:

- Rational Cherednik algebras when $G$ is a real or complex reflection group acting diagonally on $V=X \oplus X^{*}$, for $X$ the natural reflection representation, and $\kappa$ has image in $k G$ and a particular geometric form.

- Symplectic reflection algebras when $G$ acts on any symplectic vector space $V$ and $\kappa$ has image in $k G$.

- Graded affine Hecke algebras when $G$ is a Weyl group (or Coxeter group) and $\kappa$ has image in $k G$.

- Drinfeld Hecke algebras when $G$ is arbitrary and $\kappa$ has image in $k G$.

- Quantum Drinfeld Hecke algebras when $G$ is arbitrary and the nonhomogeneous relation $v_{1} \otimes v_{2}=v_{2} \otimes v_{1}+\kappa\left(v_{1}, v_{2}\right)$ is replaced by a quantum version $v_{i} \otimes v_{j}=q_{i j} v_{j} \otimes v_{i}+\kappa\left(v_{i}, v_{j}\right)$ (for a system of quantum parameters $\left.\left\{q_{i j}\right\}\right)$ and $\kappa$ has image in $k G$.

- Lie orbifold algebras when $\kappa$ has image in $V \oplus k G$ (defining a deformation of the universal enveloping algebra of a Lie algebra with group action).

Terminology arises from various settings. Drinfeld 7 originally defined these algebras for arbitrary groups and for $\kappa$ with image in $k G$. Around the same time, Lusztig (see 20, 21, for example) explored a graded version of the affine Hecke algebra for Coxeter groups. Ram and Shepler [24] showed that Lusztig's graded affine Hecke algebras are a special case of Drinfeld's construction. Etingof and Ginzburg [8] rediscovered Drinfeld's algebras for symplectic groups in the context of orbifold theory. The general case (when $\kappa$ maps to the filtered degree 1 piece of $T_{k}(V) \# G$ and $G$ acts with an arbitrary representation) is explored in [27]; see [16] in the case where the field is the real numbers. 
The original conditions of Braverman and Gaitsgory were adapted and used for determining which $\kappa$ define Drinfeld orbifold algebras, but arguments relied on the fact that the skew group algebra $S_{k}(V) \# G$ is Koszul as an algebra over the semisimple ring $k G$. (This was the approach taken by Etingof and Ginzburg [8.) Indeed, we used this theory in [27] to establish PBW conditions in the nonmodular setting. However the technique fails in modular characteristic (i.e., when the characteristic of $k$ divides the order of $G$ ).

The results in previous sections allow us to overlook the nonsemisimplicity of the group algebra in determining which quotients $\mathcal{H}_{\kappa}$ satisfy the PBW property. We consider each Drinfeld orbifold algebra as a nonhomogeneous quadratic algebra whose homogeneous version is the skew group algebra formed from a Koszul algebra and a finite group. We are now able to give a new, shorter proof of Theorem 3.1 in [27] using the methods of Braverman and Gaitsgory but in arbitrary characteristic. Thus we bypass tedious application of the Diamond Lemma 3 . (Details of a long computation using the Diamond Lemma over arbitrary fields were largely suppressed in [27.)

Decompose the alternating map $\kappa: V \otimes V \rightarrow(k \oplus V) \otimes k G$ into its linear and constant parts by writing

$$
\kappa\left(v_{1}, v_{2}\right)=\sum_{g \in G}\left(\kappa_{g}^{C}\left(v_{1}, v_{2}\right)+\kappa_{g}^{L}\left(v_{1}, v_{2}\right)\right) \otimes g
$$

for maps $\kappa_{g}^{C}: V \otimes V \rightarrow k$ and $\kappa_{g}^{L}: V \otimes V \rightarrow V$, for all $g$ in $G$. Let Alt $_{3}$ denote the alternating group on three symbols.

A version of the next result holds over fields of characteristic two, but the proposition requires a slight rephrasing and removal of the factor of 2 , so we omit that case.

Proposition 6.1 ([27, Theorem 3.1]). Let $G$ be a finite group acting linearly on a finite dimensional vector space $V$ over a field $k$ of characteristic other than 2 . The quotient algebra

$$
\mathcal{H}_{\kappa}=T_{k}(V) \# G /\left\langle v_{1} \otimes v_{2}-v_{2} \otimes v_{1}-\kappa\left(v_{1}, v_{2}\right): v_{1}, v_{2} \in V\right\rangle
$$

satisfies the Poincaré-Birkhoff-Witt property if and only if

- $\kappa$ is G-invariant,

- $\sum_{\sigma \in A l t_{3}} \kappa_{g}^{L}\left(v_{\sigma(2)}, v_{\sigma(3)}\right)\left(v_{\sigma(1)}-{ }^{g} v_{\sigma(1)}\right)=0$ in $S_{k}(V)$,

- $\sum_{\sigma \in A l t_{3}, h \in G} \kappa_{g h^{-1}}^{L}\left(v_{\sigma(1)}+{ }^{h} v_{\sigma(1)}, \kappa_{h}^{L}\left(v_{\sigma(2)}, v_{\sigma(3)}\right)\right)$

$$
=2 \sum_{\sigma \in A l t_{3}} \kappa_{g}^{C}\left(v_{\sigma(2)}, v_{\sigma(3)}\right)\left(v_{\sigma(1)}-{ }^{g} v_{\sigma(1)}\right),
$$

- $\sum_{\sigma \in A l t_{3}, h \in G} \kappa_{g h^{-1}}^{C}\left(v_{\sigma(1)}+{ }^{h} v_{\sigma(1)}, \kappa_{h}^{L}\left(v_{\sigma(2)}, v_{\sigma(3)}\right)\right)=0$,

for all $v_{1}, v_{2}, v_{3}$ in $V$ and $g$ in $G$.

Proof. Let $U=V \otimes k G$. Consider $T_{k}(V) \# G \cong T_{k G}(U)$ to be a $k G$-bimodule under the action $g_{1}\left(v \otimes g_{2}\right) g_{3}={ }^{g_{1}} v \otimes g_{1} g_{2} g_{3}$ for $g_{i}$ in $G$ and $v$ in $T_{k}(V)$. As before, we filter $T:=T_{k G}(U)$ by setting $F^{i}(T)=T^{0}+T^{1}+\ldots+T^{i}$, where $T^{i}=U^{\otimes_{k G}}{ }^{i}$ for $i>0$ and $T^{0}=k G$. Extend $\kappa$ to a map $\kappa: T_{k G}^{2}(U) \rightarrow k G \oplus U$ defined by $\kappa\left(\left(v_{1} \otimes g_{1}\right) \otimes_{k G}\left(v_{2} \otimes g_{2}\right)\right)=\kappa\left(v_{1},{ }^{g_{1}} v_{2}\right) \otimes g_{1} g_{2}$ for $v_{i}$ in $V$ and $g$ in $G$. (Note that 
$\kappa$ extends to a unique $k G$-bimodule map $\kappa: T_{k G}^{2}(U) \rightarrow k G \oplus U$ if, and only if, $\kappa$ is $G$-invariant: ${ }^{g}\left(\kappa\left(g^{-1} u, g^{-1} v\right)\right)=\kappa(u, v)$ for all $u, v$ in $V$ and $g$ in $G$.)

We set $P$ to be the generating nonhomogeneous relations parametrized by $\kappa$ : Let $P$ be the $k G$-subbimodule of $F^{2}(T)$ generated by all

$$
v_{1} \otimes_{k G} v_{2}-v_{2} \otimes_{k G} v_{1}-\kappa\left(v_{1}, v_{2}\right)
$$

for $v_{1}, v_{2}$ in $V$. We then have an isomorphism of filtered algebras, $\mathcal{H}_{\kappa} \cong T_{k G}(U) /\langle P\rangle$. Thus, $\mathcal{H}_{\kappa}$ satisfies the PBW property if and only if $T_{k G}(U) /\langle P\rangle$ exhibits the PBW type with respect to $P$ as a filtered quadratic algebra over $k G$.

We now consider the homogeneous version $A$ determined by $P$. Set $R=\pi(P)$, the $k G$-subbimodule of $T_{k G}^{2}(U)$ generated by all $v_{1} \otimes_{k G} v_{2}-v_{2} \otimes_{k G} v_{1}$ for $v_{1}, v_{2}$ in $V$. Set $A:=T_{k G}(U) /\langle R\rangle$ and note that $A \cong S_{k}(V) \# G \cong T_{k G}(U) /\langle\pi(P)\rangle$. Then as $S_{k}(V)$ is Koszul, the conditions of Theorem 5.4 apply to give explicit conditions on $\kappa$ under which $\mathcal{H}_{\kappa}$ is of PBW type. We apply the conditions as in the first proof of Theorem 3.1 of [27] without needing the extra assumption there that $k G$ is semisimple.

In the next corollary, we set the linear part of the parameter $\kappa$ to zero $\left(\kappa_{g}^{L} \equiv 0\right.$ for all $g$ in $G$ ) to recover a modular version of a result that is well known in characteristic zero (stated in [7] and then confirmed in [8] and 24]). The positive characteristic result was first shown by Griffeth 14 by construction of an explicit $\mathcal{H}_{\kappa}$-module, as in the classical proof of the PBW theorem for universal enveloping algebras of Lie algebras. (See also Bazlov and Berenstein [2] for a generalization.) Our approach yields a different proof. When $\kappa$ maps only to the group algebra (as in this case), the proof of Proposition 6.1 holds even when the characteristic of the underlying field is 2 (since for all vectors $u, v$ in $V, \beta(u \otimes v-v \otimes u)$ is just $\kappa(u, v)$ ) and the factor of 2 in the statement of the proposition is unnecessary. Thus we drop the hypothesis on the characteristic in the next corollary. Note that several authors (for example, Griffeth [14] and Balagovic and Chen [1]) study the representations of rational Cherednik algebras in the modular setting.

Corollary 6.2. Let $G$ be a finite group acting linearly on a finite dimensional vector space $V$ over an arbitrary field $k$. Suppose $\kappa: V \otimes V \rightarrow k G$ is an alternating map. The quotient algebra

$$
\mathcal{H}_{\kappa}=T_{k}(V) \# G /\left\langle v_{1} \otimes v_{2}-v_{2} \otimes v_{1}-\kappa\left(v_{1}, v_{2}\right): v_{1}, v_{2} \in V\right\rangle
$$

satisfies the Poincaré-Birkhoff-Witt property if and only if $\kappa$ is $G$-invariant and

$$
0=\sum_{\sigma \in A t_{3}} \kappa_{g}\left(v_{\sigma(2)}, v_{\sigma(3)}\right)\left({ }^{g} v_{\sigma(1)}-v_{\sigma(1)}\right)
$$

for all $v_{1}, v_{2}, v_{3}$ in $V$ and $g$ in $G$.

\section{REFERENCES}

[1] Martina Balagović and Harrison Chen, Representations of rational Cherednik algebras in positive characteristic, J. Pure Appl. Algebra 217 (2013), no. 4, 716-740, DOI 10.1016/j.jpaa.2012.09.015. MR2983846

[2] Yuri Bazlov and Arkady Berenstein, Braided doubles and rational Cherednik algebras, Adv. Math. 220 (2009), no. 5, 1466-1530, DOI 10.1016/j.aim.2008.11.004. MR2493618 (2010e:16046)

[3] George M. Bergman, The diamond lemma for ring theory, Adv. in Math. 29 (1978), no. 2, 178-218, DOI 10.1016/0001-8708(78)90010-5. MR.506890(81b:16001) 
[4] Alexander Beilinson, Victor Ginzburg, and Wolfgang Soergel, Koszul duality patterns in representation theory, J. Amer. Math. Soc. 9 (1996), no. 2, 473-527, DOI 10.1090/S0894-034796-00192-0. MR:1322847 (96k:17010)

[5] Alexander Braverman and Dennis Gaitsgory, Poincaré-Birkhoff-Witt theorem for quadratic algebras of Koszul type, J. Algebra 181 (1996), no. 2, 315-328, DOI 10.1006/jabr.1996.0122. MR,1383469 (96m:16012)

[6] José Bueso, José Gómez-Torrecillas, and Alain Verschoren, Algorithmic methods in noncommutative algebra, Mathematical Modelling: Theory and Applications, vol. 17, Kluwer Academic Publishers, Dordrecht, 2003. Applications to quantum groups. MR2006329 (2005c:16069)

[7] V. G. Drinfel'd, Degenerate affine Hecke algebras and Yangians (Russian), Funktsional. Anal. i Prilozhen. 20 (1986), no. 1, 69-70. MR831053 (87m:22044)

[8] Pavel Etingof and Victor Ginzburg, Symplectic reflection algebras, Calogero-Moser space, and deformed Harish-Chandra homomorphism, Invent. Math. 147 (2002), no. 2, 243-348, DOI 10.1007/s002220100171. MR1881922(2003b:16021)

[9] Marco Farinati, Hochschild duality, localization, and smash products, J. Algebra 284 (2005), no. 1, 415-434, DOI 10.1016/j.jalgebra.2004.09.009. MR2115022 (2005j:16009)

[10] Murray Gerstenhaber, On the deformation of rings and algebras, Ann. of Math. (2) 79 (1964), 59-103. MR0171807 (30 \#2034)

[11] M. Gerstenhaber and S. D. Schack, On the deformation of algebra morphisms and diagrams, Trans. Amer. Math. Soc. 279 (1983), no. 1, 1-50, DOI 10.2307/1999369. MR704600 (85d:16021)

[12] Murray Gerstenhaber and Samuel D. Schack, Algebraic cohomology and deformation theory, Deformation theory of algebras and structures and applications (Il Ciocco, 1986), NATO Adv. Sci. Inst. Ser. C Math. Phys. Sci., vol. 247, Kluwer Acad. Publ., Dordrecht, 1988, pp. 11-264. MR.981619 (90c:16016)

[13] Edward L. Green, Idun Reiten, and Øyvind Solberg, Dualities on generalized Koszul algebras, Mem. Amer. Math. Soc. 159 (2002), no. 754, xvi+67, DOI 10.1090/memo/0754. MR1921583 (2004b:16042)

[14] Stephen Griffeth, Towards a combinatorial representation theory for the rational Cherednik algebra of type $G(r, p, n)$, Proc. Edinb. Math. Soc. (2) 53 (2010), no. 2, 419-445, DOI 10.1017/S0013091508000904. MR2653242 (2011f:16038)

[15] Jorge A. Guccione, Juan J. Guccione, and Christian Valqui, Universal deformation formulas and braided module algebras, J. Algebra 330 (2011), 263-297, DOI 10.1016/j.jalgebra.2010.12.022. MR2774629(2012c:16089)

[16] Gilles Halbout, Jean-Michel Oudom, and Xiang Tang, Deformations of orbifolds with noncommutative linear Poisson structures, Int. Math. Res. Not. IMRN 1 (2011), 1-39, DOI 10.1093/imrn/rnq065. MR2755481(2012d:53284)

[17] U. Krähmer, "Notes on Koszul algebras," available at http://www.maths.gla.ac.uk/ ukraehmer/\#research.

[18] Huishi Li, Gröbner bases in ring theory, World Scientific Publishing Co. Pte. Ltd., Hackensack, NJ, 2012. MR2894019 (2012m:16056)

[19] L. Li, "A generalized Koszul theory and its application," Trans. Amer. Math. Soc. 366 (2014), no. 2, 931-977. MR3130322

[20] George Lusztig, Cuspidal local systems and graded Hecke algebras. I, Inst. Hautes Études Sci. Publ. Math. 67 (1988), 145-202. MR972345 (90e:22029)

[21] George Lusztig, Affine Hecke algebras and their graded version, J. Amer. Math. Soc. 2 (1989), no. 3, 599-635, DOI 10.2307/1990945. MR991016 (90e:16049)

[22] L. E. Positsel'skiı̆, Nonhomogeneous quadratic duality and curvature (Russian, with Russian summary), Funktsional. Anal. i Prilozhen. 27 (1993), no. 3, 57-66, 96, DOI 10.1007/BF01087537; English transl., Funct. Anal. Appl. 27 (1993), no. 3, 197-204. MR:1250981 (95h:16041)

[23] Alexander Polishchuk and Leonid Positselski, Quadratic algebras, University Lecture Series, vol. 37, American Mathematical Society, Providence, RI, 2005. MR2177131 (2006f:16043)

[24] Arun Ram and Anne V. Shepler, Classification of graded Hecke algebras for complex reflection groups, Comment. Math. Helv. 78 (2003), no. 2, 308-334, DOI 10.1007/s000140300013. MR.1988199 (2004d:20007) 
[25] Katsunori Sanada, On the Hochschild cohomology of crossed products, Comm. Algebra 21 (1993), no. 8, 2727-2748, DOI 10.1080/00927879308824703. MR.1222741(94k:16016)

[26] Anne V. Shepler and Sarah Witherspoon, Quantum differentiation and chain maps of bimodule complexes, Algebra Number Theory 5 (2011), no. 3, 339-360, DOI 10.2140/ant.2011.5.339. MR2833794(2012k:16026)

[27] Anne V. Shepler and Sarah Witherspoon, Drinfeld orbifold algebras, Pacific J. Math. 259 (2012), no. 1, 161-193, DOI 10.2140/pjm.2012.259.161. MR.2988488

[28] Dragoş Ştefan, Hochschild cohomology on Hopf Galois extensions, J. Pure Appl. Algebra 103 (1995), no. 2, 221-233, DOI 10.1016/0022-4049(95)00101-2. MR1358765 (96h:16013)

[29] Charles A. Weibel, An introduction to homological algebra, Cambridge Studies in Advanced Mathematics, vol. 38, Cambridge University Press, Cambridge, 1994. MR.1269324 (95f:18001)

[30] D. Woodcock, Cohen-Macaulay complexes and Koszul rings, J. London Math. Soc. (2) 57 (1998), no. 2, 398-410, DOI 10.1112/S0024610798005717. MR1644229 (99g:13025)

Department of Mathematics, University of North Texas, Denton, Texas 76203

E-mail address: ashepler@unt.edu

Department of Mathematics, Texas A\&M University, College Station, Texas 77843

E-mail address: sjw@math.tamu.edu 